\title{
Primeros veinte años de SIRGAS
}

\author{
Claudio Brunini* \\ Laura Sánchez ${ }^{* *}$
}

\begin{abstract}
This contribution describes the cornerstones of the first twenty years of the "Geocentric Reference System for the Americas" (in Spanish SIRGAS), while it pays tribute to the institutions and personalities that made it possible. In chronological order the story includes: the episodes that led to the establishment of SIRGAS and the context in those days; the establishment of SIRGAS95 as the first continentalwide reference frame compatible with the GPS, and the national densifications implemented by the Latin American countries; the actions aimed at maintaining SIRGAS, including the establishment of SIRGAS2000 and the continuously operating SIRGAS network; the efforts to establish a continental-wide vertical reference system compatible with the modern technologies; the building of capacities by the establishment of SIRGAS analysis centres in Latin American institutions; and various activities oriented to contribute to the International Association of Geodesy (IAG) "Global Geodetic Observing System", the PAIGH "2010-2020 Pan American Agenda", and the "2013-2015 PAIGH-SIRGASCPIDEA-GEOSUR Action Plan to Expedite the Development of Spatial Data Infrastructure of the Americas".

Key words: Geodesy, Reference System, SIRGAS.
\end{abstract}

\section{Resumen}

Se sintetizan los acontecimientos que jalonaron los primeros veinte años del "Sistema de Referencia Geocéntrico para las Américas" (SIRGAS), a la vez que se

* Presidente de SIRGAS, Facultad de Ciencias Astronómicas y Geofísicas de la Universidad Nacional de La Plata y Consejo Nacional de Investigaciones Científicas y Técnicas, La Plata, Argentina, correo electrónico: claudiobrunini@yahoo.com

** Vicepresidente de SIRGAS, Deutsches Geodätisches Forschungsinstitut, Alfons-Goppel-Str. 11, 80539 München, Alemania, correo electrónico: sanchez@dgfi.badw.de 
rinde un tributo a las instituciones y personalidades que los han hecho posibles. El relato, articulado en forma cronológica, abarca: los episodios que condujeron al establecimiento de SIRGAS y en el contexto en que aquellos se desarrollaron; la implementación de SIRGAS95 como primer marco de referencia continental compatible con la tecnología GPS y las densificaciones nacionales que desarrollaron los países del continente; las acciones encaminadas al mantenimiento de SIRGAS, incluyendo la medición de SIRGAS2000 y la instalación de la red SIRGAS de operación continua; los esfuerzos orientados a la definición y realización de un sistema de referencia vertical continental, compatible con las modernas tecnologías; la creación permanente de capacidades en los países de la región, abarcado la puesta en funcionamiento de centros de análisis de SIRGAS en instituciones latinoamericanas y las Escuelas SIRGAS; diversas actividades que contribuyen al cumplimento de la iniciativa "Global Geodetic Observing System" (GGOS) de la Asociación Internacional de Geodesia (IAG), a la "Agenda Panamericana 20102020" del IPGH y al "Plan de Acción Conjunto 2013-2015 para Acelerar el Desarrollo de la Infraestructura de Datos Espaciales de las Américas" del IPGH, SIRGAS, CPIDEA y GEOSUR.

Palabras clave: Geodesia, Sistema de Referencia, SIRGAS.

\section{Introducción}

En octubre de 2013 SIRGAS celebra sus primeros 20 años de existencia. Contradiciendo al maestro Carlos Gardel, que en su famoso tango "Volver" continua proclamando (desde el antiguo disco de pasta o desde el moderno MP3) que "veinte años no es nada", SIRGAS se siente orgulloso de celebrar esas dos décadas con logros importantes en su haber, con planes concretos para el futuro y con una estructura orgánica consolidada que permite afrontar confiados otras dos décadas de realizaciones.

Existe una copiosa literatura científica (parte de la cual se cita en este artículo y es accesible a través del portal de Internet de SIRGAS <www.sirgas.org $>$ ) que describe, con la rigurosidad que caracteriza a ese tipo de escritos, los avances logrados por SIRGAS en sus diferentes campos de interés y en las diferentes etapas que han sucedido en los 20 años que se cumplirán próximamente. Este artículo no abundará en detalles científicos que, como se ha dicho, se hallan extensamente tratados en la literatura específica. En lugar de ello, intentará sintetizar el camino recorrido por SIRGAS, tamizando el relato con el harnero del tiempo que filtra lo superfluo y rescata lo perdurable. Al hacerlo, intentará también rendir un tributo a las instituciones y a las personas que fueron artífices de los hechos que trascendieron el momento para incorporarse a la breve pero intensa historia de SIRGAS. 


\section{Los orígenes}

SIRGAS nació en la "Conferencia Internacional para la Definición de un Sistema de Referencia Geocéntrico para América del Sur", celebrada entre el 4 y el 7 de octubre de 1993, en Asunción (Paraguay), con el objeto de enfrentar la problemática que el nombre de la conferencia enunciaba y que generó el acrónimo que identificó a la organización que se estableció para enfrentarla (Kumar 1993, Drewes 1995). La Conferencia, que fue convocada por la Asociación Internacional de Geodesia (en inglés IAG), el Instituto Panamericano de Geografía e Historia (IPGH) y la Defence Mapping Agency ${ }^{1}$ (DMA) de los Estados Unidos de América (EUA), fue atendida por delegados de nueve países de la región: Argentina, Brasil, Colombia, Chile, Ecuador, Guyana Francesa, Paraguay, Uruguay y Venezuela; a ellos se sumaron conferencistas provenientes de Alemania, Canadá, Dinamarca, España y Estados Unidos (Figura 1). La mayoría de los sudamericanos habían sido delegados por los institutos geográficos y los restantes provenían de universidades. Esa presencia institucional se mantendría a lo largo de los años, proveyendo el basamento sobre el que creció y se consolidó SIRGAS. Es justo remarcar desde el principio que a esa construcción contribuyeron además, en forma permanente, el IPGH, la IAG y el Deutsches Geodätisches Forschungsinstitut (DGFI) de Alemania, y, durante la primera mitad de la vida de SIRGAS, la DMA.

La Conferencia estableció una organización compuesta por un comité de proyecto, un consejo científico y dos grupos de trabajo (GT) que se denominaron "I - Sistema de Referencia" y "II - Datum Geocéntrico": el primero con la responsabilidad de materializar el marco de referencia continental y el segundo con la de extenderlo en cada país. El comité quedó integrado por representantes de las tres instituciones que habían convocado la Conferencia — que a partir de ese momento se convirtieron en patrocinadoras del proyecto- y de todos los países presentes en la misma. Su presidencia recayó sobre Luiz Paulo Souto Fortes, del Instituto Brasileiro de Geografia e Estatística (IBGE, Brasil); y las de los GT I y II sobre Melvin Hoyer, de la Universidad del Zulia (Luz, Venezuela), y Walter Subiza, del Servicio Geográfico Nacional (SGM, Uruguay), respectivamente. Los países que no se hallaban representados en la Conferencia fueron invitados a integrarse al proyecto y para comienzos de 1994 todos, salvo Surinam, habían nombrado a sus representantes en el comité. Para la época, las instituciones patrocinadoras habían designado a sus representantes: Hermann Drewes, del DGFI, por la IAG; Fernando Galban, del IGM argentino, por el IPGH; y James Slater, de y por la DMA. 


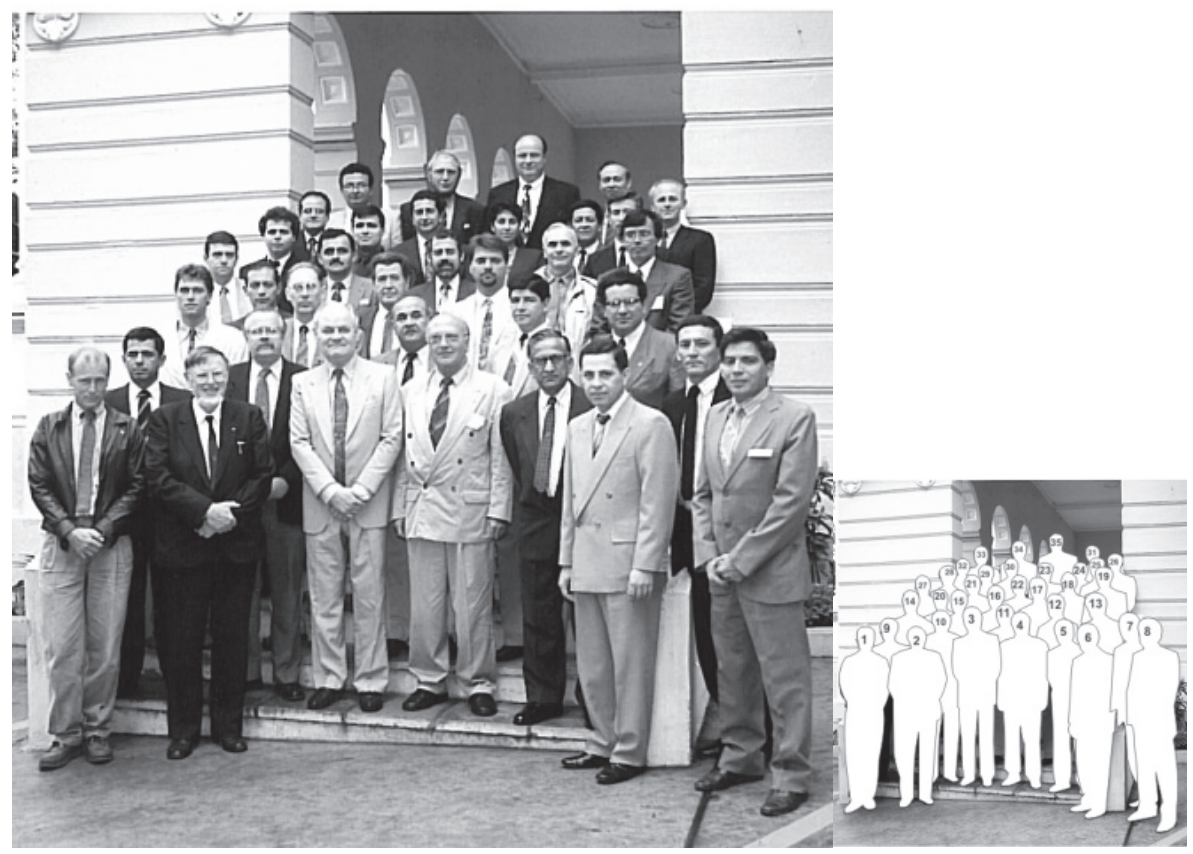

Figura 1. Participantes de la Conferencia fundacional de SIRGAS: 2) K. Poder (Dinamarca); 3) R. Rodríguez (IPGH); 4) W. Torge (IAG); 5) Muneendra Kumar (DMA); 6) L. Centurión (Paraguay); 10) E. Pallejá (Argentina); 13) S. Bruni (Brasil); 14) H. Fagard (Guyana Francesa); 16) J. L. Caturla (España); 17) L. P. Souto Fortes (Brasil); 18) M. Pinch (Canadá); 22) H. Drewes (Alemania); 23) S. Arciniegas (Ecuador); 24) A. Gonzáles (Colombia); 25) O. Cifuentes (Chile); 26) A. Stahlschmidt (Argentina); 27) W. Subiza (Uruguay); 28) E. Fonseca Junior (Brasil); 30) E. Elinan (Estados Unidos); 31) J. Konig (Argentina); 33) M. Hoyer (Vnezuela); 33) J. Napoleón Hernández (Venezuela); 34) G. Seeber (Alemania); los otros participantes, que los autores no logran identificar en la foto, son: H. Beattie, D. Lehman, J. Richardson y R. Zebell (Estados Unidos); B. Fernández Chaparro (Colombia); O. Niño (Venezuela); y J. L. Alder, G. Rodríguez, A. Irala, G. Alarcon, P. Andino, D. Arias, M. A. Bello, A. Coronel, L. Irigoitia, J. E. Martínez, M. A. Nuñez, C. Orue y B. Peña (Paraguay). Copia de la fotografía con la información adjunta fue cedida amablemente por R. Rodríguez.

\section{El contexto}

El gran interés que la Conferencia de 1993 despertó en los institutos geográficos sudamericanos anticipaba que la temática tocaba una fibra sensible de esas instituciones. Ello sería rotundamente confirmado en los años venideros. Por aquellos días, el Sistema de Posicionamiento Global (en inglés GPS) se había constituido en la herramienta preferida para "georreferenciar" los levantamientos cartográficos. 
"Georreferenciar" no era el único neologismo que repicaba con fuerza en aquellos días: otros avances tecnológicos acechaban detrás de las expresiones "Sistema de Información Geográfica" (en inglés GIS) o "Infraestructura de Datos Espaciales" (IDE). El poder de las computadoras personales se multiplicaba al tiempo que se reducían sus costos, la información "analógica" libraba una batalla desigual contra la "digital" y la telaraña global de la Internet popularizaba las siglas "www".

Los autores de este artículo no desean conjeturar acerca de si aquellos cambios tecnológicos fueron propiciadores de las transformaciones políticas, económicas y sociales que se desarrollaron en Sudamérica durante la década de los noventa; o si, por el contrario, la tecnología fue otro emergente de aquellas transformaciones. Lo cierto es que aquella conjunción de factores multiplicó el valor de los recursos territoriales de los países y, con ello, la necesidad de información espacial actualizada y confiable. Los autores tampoco desean conjeturar acerca de cuan soberana fue la decisión de los países sudamericanos, de contraer deudas públicas con organismos financieros internacionales para afrontar los costos que implicaba la modernización cartográfica. Lo cierto es que, en mayor o menor medida, todos ellos contrajeron deudas cuantiosas para enfrentar aquella indispensable modernización.

Aquel contexto patentizó la desactualización que habían acumulado las infraestructuras geodésicas de nuestros países (Cadess et al., 1993). Cada uno a su modo, todos habían invertido esfuerzos ingentes para establecer redes geodésicas convencionales, horizontales y verticales, que eran imprescindibles para cartografiar sus vastos territorios y llevar el progreso a las regiones más distantes. Pero aquellas inversiones de la primera mitad del siglo XX habían ido menguando, hasta casi detenerse en la década de los sesenta. Los esfuerzos para establecer el South American Datum (SAD) — una red geodésica de cobertura continental que permitiera la integración de todas las redes nacionales - chocaban contra la extensión del territorio y la impenetrabilidad de muchas regiones. Y la unificación que no se había logrado a escala continental, tampoco se había logrado a escala nacional pues en los países coexistía una diversidad de redes que se habían establecido "provisoriamente" hasta que la red geodésica nacional llegase a esa región.

Para la década de los noventa, las redes geodésicas que habían sido excelentes en su tiempo, se hallaban incompletas, desactualizadas y resultaban incompatibles con las modernas tecnologías "geomáticas" (he allí otro neologismo acuñado en la época). Ese era el contexto que abonaba el terreno donde cayó la semilla de un proyecto destinado a establecer una red geodésica continental, moderna, precisa y compatible con el GPS. Una anécdota que describe el clima del momento con precisión y economía de palabras les fue narrada a los autores por el principal artífice de aquellos años iniciáticos de SIRGAS: cuenta Hermann Drewes que pocos meses antes de la Conferencia en Paraguay, más precisamente en junio de 1993, escribió cartas (el correo electrónico no era aún tan popular) a los institutos geográficos 
invitándolos a aunar esfuerzos para la medición con GPS de una red continental y consultándolos sobre la oportunidad en que podrían abordar la empresa (Figura 2). La respuesta no se hizo esperar: todos aceptaron la invitación y lo urgieron para que la empresa se acometiera de inmediato.
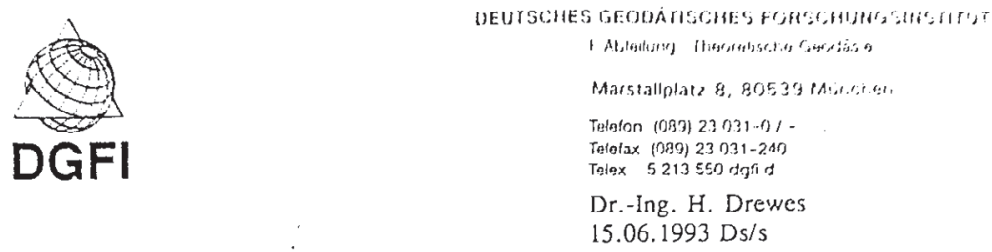

Sistema de Referencía por GPS en América del Sur (SIRGAS)

Muy estimado colega,

desde hace unos años se están efectuando varios proyectos geodésicos en América del Sur utilizando el sistema de posiciunamiento global (Global Positioning System - GPS). Unos de estos proyectos se basan en una cooperación internacional con grupos científicns do o Europa y EE.UU. Los objectivos de los proyectos snn arestre con exactitud de mas los geodinámicas para el mnţél tétérencia de la orbita y distancias de $100 \mathrm{~km}$. Como meuros tormidad de los sistemantizar la precisión de $1 \mathrm{~cm}$ en dis desviaciones de varios de la menos $10 \mathrm{~cm}$ para garantuales en América del a este sistema para aprovectarse sisternas de referencia actuales

del sistema ITRF, presisión de GPS

pel sistema
Erision de GPS.
ristás explicarme sus ideas para integrarias en la planificación de la campaña.

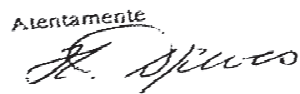

Dr.-Ing. H. Drewes

Figura 2. Fragmentos de la invitación dirigida por H. Drewes a los institutos geográficos de Sudamérica en junio de 1993.

\section{Los primeros pasos}

La primera definición relevante del proyecto SIRGAS surgió en la Conferencia fundacional de 1993: el sistema de referencia que se materializaría en Sudamérica sería el "Sistema de Referencia Terrestre del Servicio Internacional de Rotación de la Tierra" (en inglés ITRS). ${ }^{2}$ No está de más recordar que aquella decisión fue precedida por largos y fragorosos debates en torno a la conveniencia de usar ese sistema, que por entonces era un emergente impulsado por la comunidad científica internacional, o apoyarse en el ya consolidado WGS84 (World Geodetic System, 
1984), que era el sistema establecido por la DMA y utilizado para las efemérides transmitidas de los satélites GPS. Los hechos por venir confirmarían con creces el valor de aquellos debates y lo certero de la decisión alcanzada. Aquella conducta fue la marca bautismal de SIRGAS, que siempre se ha caracterizado por poner su mira en la vanguardia geodésica internacional y tomar sus decisiones democráticamente, con la participación activa de los actores sudamericanos, luego de dirimir las diferencias de opiniones a través del debate abierto y constructivo.

Es verdad que la decisión tomada por la Conferencia de 1993 fue muy relevante, pero no menos cierto es que nadie la recordaría 20 años después si su letra no se hubiera transformado en un hecho sólido, concreto y materializado en el terreno: el marco de referencia SIRGAS95; el primer marco de referencia geocéntrico global, de alcance continental y compatible con el GPS, que tuvo la América del Sur. Su gesta se inició durante la primera reunión del GT II, celebrada entre el 20 y el 22 de abril de 1994, en Bogotá, más precisamente, en la sede del Instituto Geográfico Agustín Codazzi (Colombia), donde se estableció cómo se distribuirían las estaciones que compondrían la futura red geodésica. Aquella planificación, que intentaba conciliar aspiraciones (establecer una red tan densa como fuera posible, con puntos distribuidos equitativamente en todos los países, en sitios coincidentes con los puntos fundamentales de las redes pre-existentes, etc.) con limitaciones (disponibilidad de equipos de medición, capacidad para procesar las mediciones, infraestructura disponible en los sitios de medición, etc.), condujo al diseño de una red con 48 estaciones distribuidas de la siguiente manera: seis en Argentina, cuatro en Bolivia, diez en Brasil, cuatro en Chile, cuatro en Colombia, tres en Ecuador, uno en Guyana, uno en Guyana Francesa, dos en Paraguay, cuatro en Perú, uno en Surinam, dos en Uruguay, cuatro en Venezuela y dos en países insulares. La red que se materializaría poco tiempo después no diferiría mucho de aquella planificación.

La segunda reunión del GT II y la primera del GT I tuvieron lugar entre el 24 y el 28 de octubre de 1994, en esta ocasión en La Plata (Argentina), donde se celebraba la 18 Reunión Científica de la Asociación Argentina de Geofísicos y Geodestas. Las discusiones, que fueron muy extensas, abarcaron todos los temas relacionados con la organización de la campaña de medición GPS, que se llevaría a cabo simultáneamente en todo el continente, durante diez días ininterrumpidos, arrancando a las $0^{\mathrm{h}}$ de Tiempo Universal del 26 de mayo de 1995. Ningún detalle escapó al escrutinio de los expertos allí reunidos, desde las órbitas de los satélites hasta el tipo de monumento aceptable para montar las antenas GPS.

\section{El primer "gran logro"}

De acuerdo con lo planificado, las mediciones GPS se realizaron durante las 24 horas de los días comprendidos entre el 26 de mayo y el 4 de junio de 1995, en forma simultánea, en los 58 sitios distribuidos en 11 países sudamericanos (Figura 3). 
Cada país designó una institución responsable de colectar las mediciones hechas en su territorio y remitirlas a los bancos de datos que, para garantizar redundancia, fueron dos: uno administrado por el DGFI y el otro por el Instituto Brasileiro de Geografía y Estadística (IBGE). Para mediados de agosto, la totalidad de los datos se hallaba disponible en ambos bancos, culminando así la fase de medición de la red. La concreción exitosa de esa tarea, en la que participaron unas 30 instituciones de América del Sur, Alemania y Estados Unidos, fue otra impronta del proyecto SIRGAS: el trabajo coordinado en pro de metas colectivas.

La red fue calculada por dos centros de procesamiento que trabajaron independientemente y con diferentes programas de cálculo: uno en el DGFI, utilizando el programa Bernese, ${ }^{3}$ y el otro en la DMA — que por entonces se había convertido en la National Imagery and Mapping Agency (NIMA) - , utilizando el programa GIPSY-OASIS. ${ }^{4}$ Diferencias menores que un centímetro entre las dos soluciones engendraron extensas discusiones durante las reuniones que ambos GT mantuvieron en Santiago (Chile), entre el 5 y el 9 de agosto de 1996, en ocasión de celebrarse el IV Congreso Internacional de Ciencias de la Tierra que convocaba el Instituto Geográfico Militar de ese país.

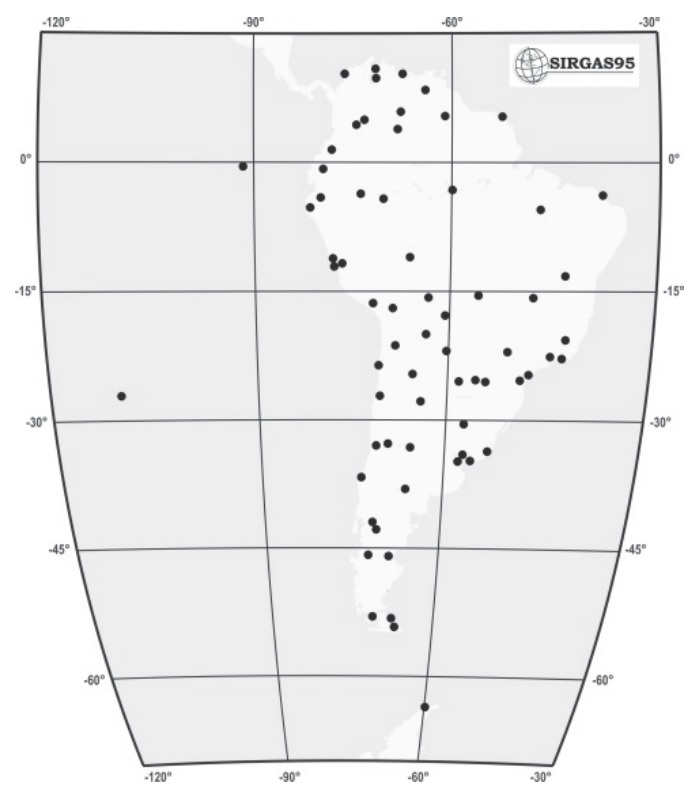

Figura 3. Los 58 sitios que conformaron la red SIRGAS95 ubicados en 11 países sudamericanos. 
Los GT I y II volvieron a reunirse entre el 8 y el 11 de abril de 1997, en la Isla Margarita (Venezuela), con el mandato de zanjar las discrepancias y generar la solución definitiva de la red, cosa que se logró, siempre por el camino del debate y el consenso, mediante la combinación de las dos soluciones y su vinculación al Marco de Referencia Terrestre Internacional vigente en ese momento, el ITRF94. Es justo reconocer aquí la labor sobresaliente que cumplieron los responsables de los cálculos: Klaus Kanniuth en DGFI y James Slater en NIMA; y los principales impulsores de las discusiones que condujeron a la solución unificada: Hermann Drewes, de DGFI, y Muneendra Kumar, de NIMA (Fortes et al., 1995; Drewes et al., 1997). Aquella solución unificada fue oficialmente aceptada por SIRGAS durante las reuniones que se celebraron en Río de Janeiro (Brasil), entre el 3 y el 9 de septiembre de 1997, en concordancia con la Asamblea Científica de la IAG (SIRGAS 1997; Kaniuth et al., 1998). ¡La precisión de las coordenadas finales de SIRGAS95 resultó mejor que muy pocos milímetros!

\section{Las densificaciones nacionales de SIRGAS}

Las reuniones que los GT de SIRGAS mantuvieron en agosto de 1996, en Chile, cobijaron también intensos debates sobre el modo en que cada país densificaría la red continental para facilitarles la accesibilidad a los usuarios. Aquellos debates convergieron a dos opciones: 1) NIMA tomaría a su cargo el reajuste a SIRGAS95 de las antiguas redes geodésicas de todos los países; o 2) cada país remediría su red geodésica con GPS y ajustaría las nuevas mediciones a SIRGAS95. La escogida fue la segunda opción, que privilegiaba el establecimiento de redes medidas con la nueva tecnología y la participación activa de las instituciones sudamericanas en la densificación de SIRGAS95.

Los años venideros vieron expandirse las redes geodésicas que densificaban a SIRGAS95 (y posteriormente a SIRGAS2000) en los diferentes países, medidas y calculadas por los institutos geográficos con la asistencia científica de algunas universidades latinoamericanas, de instituciones de los Estados Unidos y, sobre todo, del DGFI (Drewes et al., 1998; Moirano et al., 1998; Brunini et al., 2000; Tremel et al., 2001; Sánchez 2004; Costa y Lima, 2005; Martínez y Sánchez, 2009; Echalar y Sánchez, 2010; Figueroa et al., 2010; Zurita et al., 2010). Esas redes eran, al igual que SIRGAS95, "pasivas", significando con ello que estaban materializadas por monumentos cuyas coordenadas se calculaban a partir de mediciones GPS colectadas durante un cierto intervalo (generalmente, varios días). Como se verá de inmediato, aquellas redes pasivas fueron dejando paso a las "activas", formadas por monumentos sobre los que funciona un receptor "GNSS continuo". Las siglas GNSS (Global Navigation Satellite Systems) aluden a receptores que poseen la capacidad de recibir las señales de los sistemas GLONASS, Galileo, etc., además 
del GPS; y la palabra "continuo" alude a que esos receptores permanecen activos las 24 horas de los 365 días del año.

Los diferentes países de la región fueron migrando progresivamente a SIRGAS sus antiguos marcos de referencias nacionales. En la actualidad, SIRGAS ha sido adoptado oficialmente por 14 países: Bolivia, Brasil, Chile, Colombia, Costa Rica, Ecuador, El Salvador, Guyana Francesa, Guatemala, México, Panamá, Perú, Uruguay y Venezuela. Las redes nacionales que densifican a SIRGAS comprenden actualmente más de 3,200 puntos, 272 de los cuales son activos (Figura 4). Otros países de la región encaminan esfuerzos hacia la adopción oficial de SIRGAS, siguiendo la recomendación promulgada por la Organización de las Naciones Unidas (ONU), a través de su VII Conferencia Cartográfica de las Américas, celebrada entre el 22 y el 26 de enero de 2001, en Nueva York (Estados Unidos).

\section{El sistema de referencia vertical}

SIRGAS95 proporcionaba un marco de referencia inmejorable para las tres coordenadas que se determinan con los GNSS: la longitud, la latitud y la altura geométrica cuya superficie de referencia es el elipsoide. Pero ni los GNSS, ni el marco de referencia SIRGAS, son apropiados para determinar la altura física, cuya magnitud y superficie de referencia son definidas por el campo de gravedad de la Tierra. Para permitir el desarrollo de las obras de ingeniería - especialmente las que implican flujos de agua - , los países de la región establecieron redes verticales — también llamadas de nivelación-, que complementaban a sus antiguas redes geodésicas. Siguiendo la convención de la época, que utilizaba el geoide como superficie de referencia para las alturas físicas, y se apoyaba en el nivel medio del mar para materializarlo, utilizaron mareógrafos en las costas oceánicas para establecer el "cero" de sus sistemas verticales y el laborioso procedimiento geodésico de la nivelación para extender las redes de nivelación hacia el interior del continente.

En los días de SIRGAS, la Geodesia había avanzado lo suficiente para anticipar que las redes verticales de los diferentes países sudamericanos eran inconsistentes entre sí y que su precisión era insuficiente para las nuevas tecnologías (Drewes 2001). Varias razones sustentaban aquel anticipo, entre las más importantes: los "ceros" determinados con los mareógrafos podían apartarse significativamente (desde algunos decímetros hasta pocos metros) del nivel medio del mar - y en consecuencia, del geoide-, a causa de procesos dinámicos locales y regionales que tienen lugar en los océanos; y los errores causados por las irregularidades del campo de gravedad en las alturas niveladas no habían sido corregidos cuando se establecieron las redes de nivelación de los países latinoamericanos. Las vinculaciones de las redes de nivelación de países limítrofes, que SIRGAS concretaría años después, certificaron aquella presunción y cuantificarían la magnitud de las discrepancias, que iban desde pocos centímetros a algunos metros (Figura 5). 


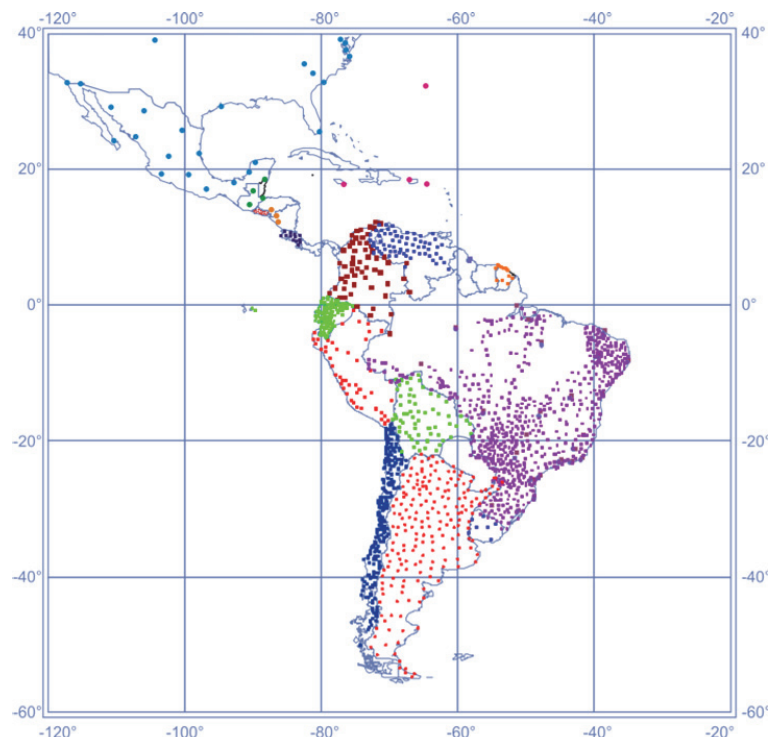

Figura 4. Densificaciones nacionales de SIRGAS mediante redes pasivas y activas.

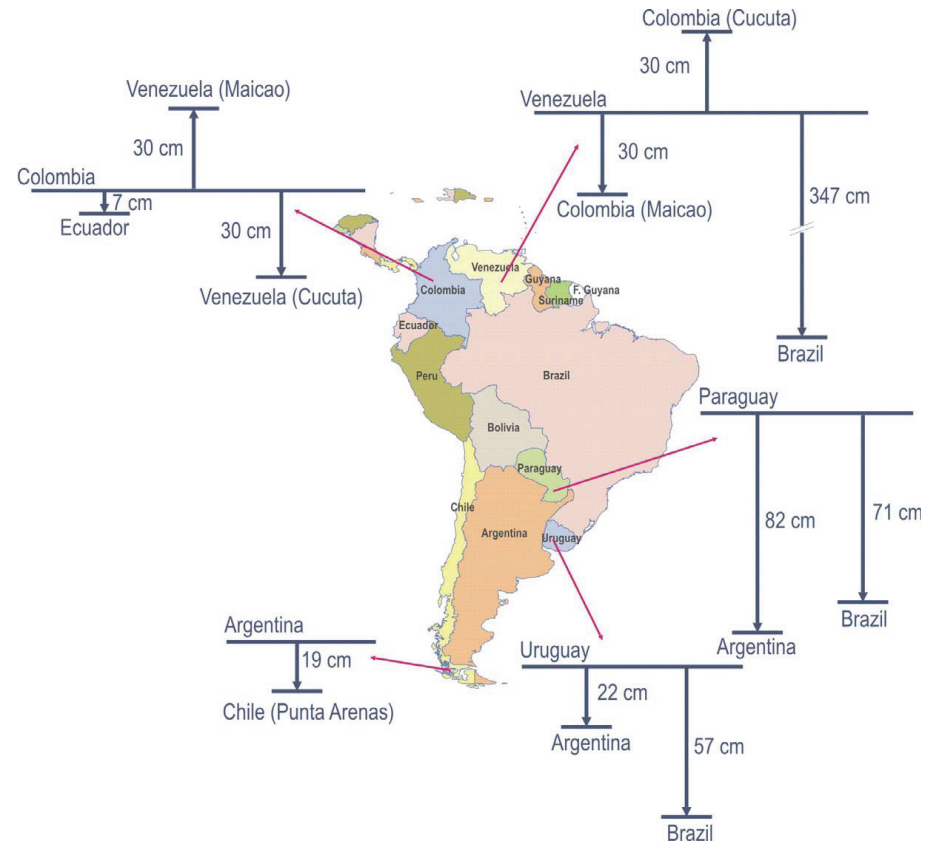

Figura 5. Discrepancias entre las redes de nivelación de países vecinos determinadas en varias vinculaciones fronterizas. 


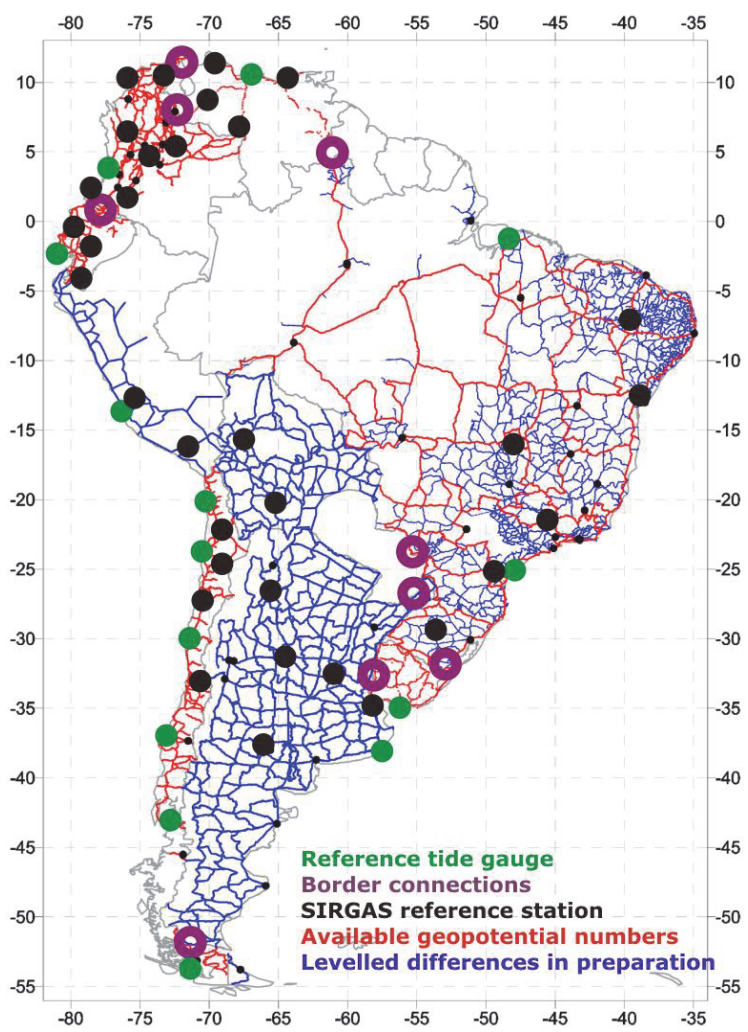

Figura 6. Información disponible en los centros de datos del Grupo de Trabajo III -Datum Vertical- de SIRGAS.

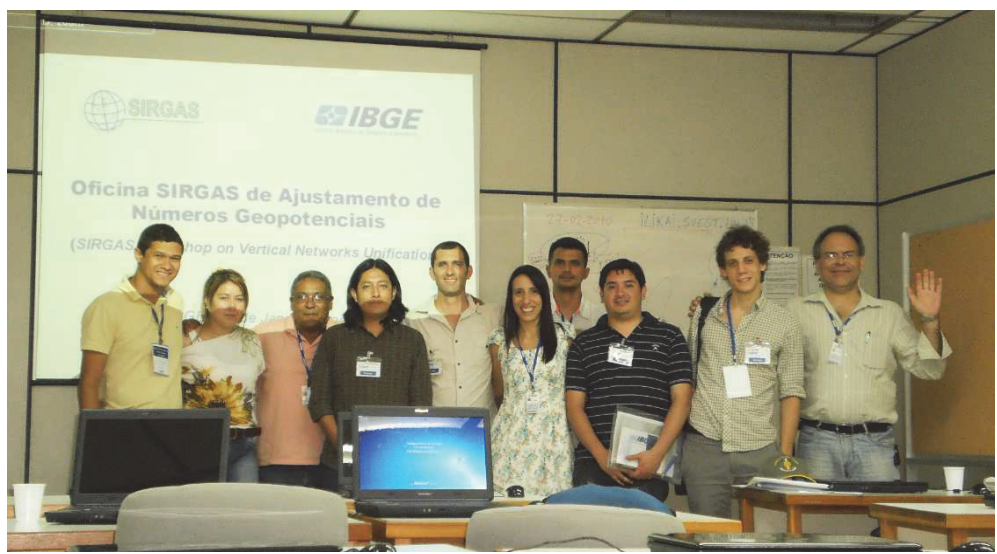

Figura 7. Participantes del Segundo Taller del Grupo de Trabajo III de SIRGAS, celebrado en Río de Janeiro (Brasil) entre el 3 y el 6 de diciembre de 2012. 


\section{EI Grupo de Trabajo III de SIRGAS}

SIRGAS diagnosticó prematuramente esos problemas y para enfrentarlos estableció un tercer GT denominado "III - Datum Vertical". Su creación se decidió durante las reuniones de 1997, en Río de Janeiro, y su presidencia recayó en Roberto Texeira Luz, del IBGE (Brasil). A 16 años de su creación, resulta ineludible admitir que el GT III no ha logrado avanzar tan rápidamente como los otros dos. Pero ese balance, que a primera vista suena desalentador, se vuelve mucho más benigno cuando se toma en cuenta la extrema laboriosidad de la tarea que enfrenta este GT.

Los fundamentos conceptuales para establecer un nuevo marco de referencia vertical en América Latina se discutieron intensamente durante la ya mencionada reunión de 1997, en el Primer Taller del GT III, realizado en Santiago (Chile), entre el 10 y el 14 de agosto de 1998, en ocasión de celebrarse el V Congreso Internacional de Ciencias de la Tierra, convocado por el IGM de ese país (Drewes et al., 2002; Freitas et al., 2002; Luz, 2008; Sánchez, 2007; Sánchez, 2009). Ese marco conceptual evolucionó con los años, pero su substancia no cambió significativamente. Las reuniones que se sucedieron en los años venideros cobijaron intensas discusiones del GT III, pero estas se orientaron principalmente a coordinar e impulsar las tareas necesarias para llevar a la práctica aquel basamento conceptual. Para ello, era necesario: determinar el nivel de referencia ( $\mathrm{W}_{0}$ en la jerga geodésica) mediante mediciones globales de altimetría satelital oceánica y de mareógrafos controlados con GNSS; vincular las redes de nivelación de alta precisión de los diferentes países entre sí, a los puntos SIRGAS y a los mareógrafos de referencia; compensar la red vertical continental, incluyendo las correcciones gravimétricas.

Las tareas orientadas a determinar $\mathrm{W}_{0}$, que fueron abordadas en el DGFI, avanzaron con relativa velocidad (lo que no significa con facilidad) y con éxitos resonantes como, por ejemplo, impulsar la revisión del valor convencional utilizado por la IAG y por la Unión Astronómica Internacional (en inglés IAU), para reducir la discrepancia de aquel valor con el determinado por SIRGAS. La laboriosa determinación del $\mathrm{W}_{0}$ involucró el análisis de largas series de datos de diferentes satélites altimétricos, la evaluación de los modelos globales de geoide producidos por las misiones espaciales de la última década y el análisis de una red global de mareógrafos controlados con GPS (Sánchez, 2007; Sánchez, 2008).

Las vinculaciones verticales avanzaron con menos velocidad, condicionadas por el tiempo y los recursos que los institutos geográficos pudieron dedicar a la tarea que se agregaba a su ya recargada agenda laboral. En la actualidad se realizan los últimos esfuerzos para completar la compilación de los desniveles y la gravedad que los diferentes países midieron en décadas de esforzado trabajo (Figura 6). Un simple detalle ilustra la dificultad de la empresa: la mayor parte de la información requerida se halla asentada en antiguas libretas de campo que deben trascribirse 
manualmente al formato digital. Para compilar y procesar la información vertical, SIRGAS dispone de dos centros de datos y análisis, el IBGE y el DGFI, los cuales contienen réplicas idénticas de la información disponible y adelantan simultáneamente los análisis del caso, de modo que haya la redundancia necesaria para obtener resultados confiables.

Un impulso trascedente a la tarea de este GT se concretó durante el "Segundo Taller del GT III de SIRGAS", que tuvo lugar entre el 3 y el 6 de diciembre de 2012, en Río de Janeiro (Brasil), con el apoyo del IBGE, del IPGH y de la IAG. Allí se reunieron delegados de los institutos geográficos de Argentina, Brasil, Chile, Ecuador, Perú y Uruguay (Figura 7). Bajo la supervisión del Presidente del GT - Roberto Texeira Luz - y con la asistencia de la responsable del banco de datos del IBGE - Nívea Régis di Maio—, se llevó a cabo el primer ajuste conjunto de las redes de nivelación de los países mencionados. Estas actividades fueron extendidas a Bolivia, Colombia y Perú mediante visitas porteriores del presidente del GT III a esos países.

\section{SIRGAS en la transición del siglo $\mathrm{XX}$ al siglo XXI}

El cambio de milenio fue una etapa que bien podría caracterizarse por las transformaciones sociales, económicas y políticas que experimentaron los países de América Latina, así como por avances científicos y tecnológicos que se produjeron en el mundo. Sin pretensión de - y sin la capacidad para - analizar semejantes procesos, los autores se atreven a llamar la atención sobre el impacto que tuvo en la vida de SIRGAS — que se desarrolló a caballo de ese cambio de milenio-, la conceptualización del territorio como un bien imprescindible para el desarrollo social y económico y para la sustentabilidad política de las naciones y la toma de conciencia colectiva sobre su vulnerabilidad.

Actuando juntos, ese concepto y esa conciencia multiplicaron la necesidad de información territorial que los gobiernos requerían para implementar sus políticas sociales y económicas. Y esa multiplicación no operó solamente sobre la cantidad, sino también sobre la calidad de la información (mayor precisión, compatibilidad regional, global y con los satélites, soporte digital, disponibilidad inmediata, actualización permanente, facilidades de intercambio, formatos estandarizados, etc.). Los tomadores de decisión dejaron de imaginar a la información territorial como una hoja de papel, inmutable en el tiempo, a la que bastaba consultar una vez para informarse de todo lo que contenía; para visualizarla como un ente formado por bits de computadora, en constante mutación y capas de viajar instantáneamente a través de la Internet. Y de ese ente esperaban respuestas para los problemas clásicos, como los que plantea el catastro o la ingeniería topográfica; pero también para los novedosos, como los que plantean los desastres naturales o el cambio climático. 
SIRGAS se gestó y evolucionó en ese contexto cambiante y demostró una gran capacidad de innovación para responder a las demandas, cada vez más exigentes, que planteaban las necesidades sociales, políticas y económicas de la región, y una gran versatilidad para aplicar los avances científicos y tecnológicos internacionales a la solución de problemas regionales (Drewes, 1999 y 2003).

SIRGAS nació en 1993 para dotar a América del Sur de un marco de referencia compatible con la ciencia y la tecnología de finales de la centuria pasada. Ese marco de referencia era imprescindible para soportar el desarrollo de la IDE de las Américas, que también emergía por la época. La concreción de esa meta llegó de la mano de SIRGAS95, cuya oficialización por parte de los países sudamericanos comenzó en 1997. Para entonces, las demandas sobre SIRGAS95 excedían las que planteaban las IDE, porque ya se avizoraba que para articular mejor la convivencia entre el hombre, con su necesidad creciente de bienestar, y el planeta, con su capacidad limitada para satisfacer esa necesidad, era indispensable disponer de mejor información de base geodésica.

Esto implicaba comprender mejor los cambios que experimenta el planeta, sintetizados en la expresión "cambio global" y, para ello, era indispensable medir señales muy pequeñas, que se desabollan con extrema lentitud (solo por dar un ejemplo: se estima que el nivel medio de los océanos del planeta está aumentando con una velocidad de aproximadamente $3 \mathrm{~mm} / \mathrm{a}$ en respuesta al calentamiento global). Para cuantificar esas señales se hizo necesario llevar la precisión de los instrumentos de medición a límites que nunca antes habían sido imaginados; y para que los resultados que emergían de esas mediciones fueran confiables se hizo evidente la necesidad de repetir las mediciones constantemente y a lo largo de muchos años. Pero si lo que se deseaba cuantificar era una señal de cambio, era necesario referir las mediciones a "algo" que no cambiara dentro de límites muy estrictos y durante muchos años. Pero ese "algo", que en la escala planetaria era el ITRF y en la escala regional era SIRGAS95, estaba materializado sobre la superficie del planeta... ique cambia permanentemente!

En otras palabras, los 58 monumentos geodésicos que materializan el marco de referencia SIRGAS95 (Figura 1) se hallan rígidamente anclados al terreno y se mueven junto con este, en respuesta a una variedad de procesos geofísicos que afectan al planeta (procesos tectónicos globales, regionales y locales, efectos de carga producidos por los océanos, la atmósfera, los hilos o las aguas superficiales y subterráneas, actividad sísmica y volcánica, etc.). Bastan pocos años para que esos movimientos, que se desarrollan con velocidades de entre algunos $\mathrm{mm} / \mathrm{a}$ y varios $\mathrm{cm} / \mathrm{a}$, modifiquen las coordenadas de los puntos SIRGAS95, causando errores que superan la exactitud de las mismas... ique es de muy pocos $\mathrm{mm}$ ! Y ello cuando no ocurre un terremoto que, en unos cuantos segundos puede provocar desplazamientos superiores al metro. 


\section{EI mantenimiento de SIRGAS}

Un marco de referencia útil para estudiar el cambio global no puede ser establecido de una vez y para siempre. Es necesario remedirlo periódicamente, para evaluar los cambios que afectan a las coordenadas de los puntos de referencia. Esos cambios deben ser descontados de las señales de cambio global que se desean estudiar. Ese concepto era claro para los miembros fundadores de SIRGAS, quienes en la conferencia de 1993 enunciaron el objetivo de establecer el marco de referencia y lo ligaron indisolublemente con el de su mantenimiento.

Las acciones concretas orientadas al mantenimiento de SIRGAS se iniciaron en las reuniones del 22 y 23 de julio de 1999, durante la 22a Asamblea Científica de la Unión Internacional de Geodesia y Geofísicas (en inglés IUGG), en Birmingham (Reino Unido de Gran Bretaña). Allí se programó la segunda medición con GPS de la red SIRGAS, lo cual se concretó entre el 10 y el 19 de mayo de 2000. Para entonces, el número de sitios de medición había aumentado de 58 a 184 (varios de ellos ya estaban ocupados con receptores GPS de medición continua) y su distribución se había expandido de América del Sur a América Central, al Caribe y a Norteamérica. Al objetivo primordial del mantenimiento de SIRGAS se habían agregado otros orientados a satisfacer las demandas del recientemente creado GT III (Luz et al., 2002). Estos implicaban medir en los mareógrafos de referencia de América del Sur y puntos fronterizos que permitían la conexión de las redes de nivelación de países vecinos (Figura 8).

La red SIRGAS2000 fue calculada por tres centros de procesamiento bajo las responsabilidades del DGFI, el IBGE y la Bayerische Kommission für die Internationale Erdmessung (BEK) de Alemania (Kaniuth et al., 2002a; Drewes et al., 2005). Los dos primeros utilizaron el programa Bernese y el tercero el programa GIPSY-OASIS. Las reuniones que se celebraron en los años subsiguientes permitieron evaluar los avances en el procesamiento de las mediciones. Finalmente, en Santiago de Chile, entre el 21 y el 22 de octubre de 2002, en el marco del VII Congreso Internacional de Ciencias de la Tierra que convocara IGM de ese país, se discutieron los resultados de los tres procesamientos. Las discrepancias entre ellos resultaron de pocos milímetros. Para entonces, la confianza de SIRGAS en sus centros de procesamiento se hallaba tan consolidada que se habilitó al DGFI a combinar las tres soluciones individuales, generar la solución definitiva de SIRGAS 2000, vinculada al ITRF2000, y proceder a su difusión como producto oficial de SIRGAS sin aguardar a las discusiones que se darían en las reuniones venideras. Como modesto reconocimiento al trabajo realizado se mencionan aquí a los responsables de los centros de procesamiento: Klaus Kanniuth en DGFI, Sonia Alves Costa en IBGE y Christof Völksen en BKE. También es digno recordar las enriquecedoras discusiones científicas que sostuvieron, una vez más en la reunión de Chile, Hermann Drewes de DGFI y Muneendra Kumar de NIMA. 


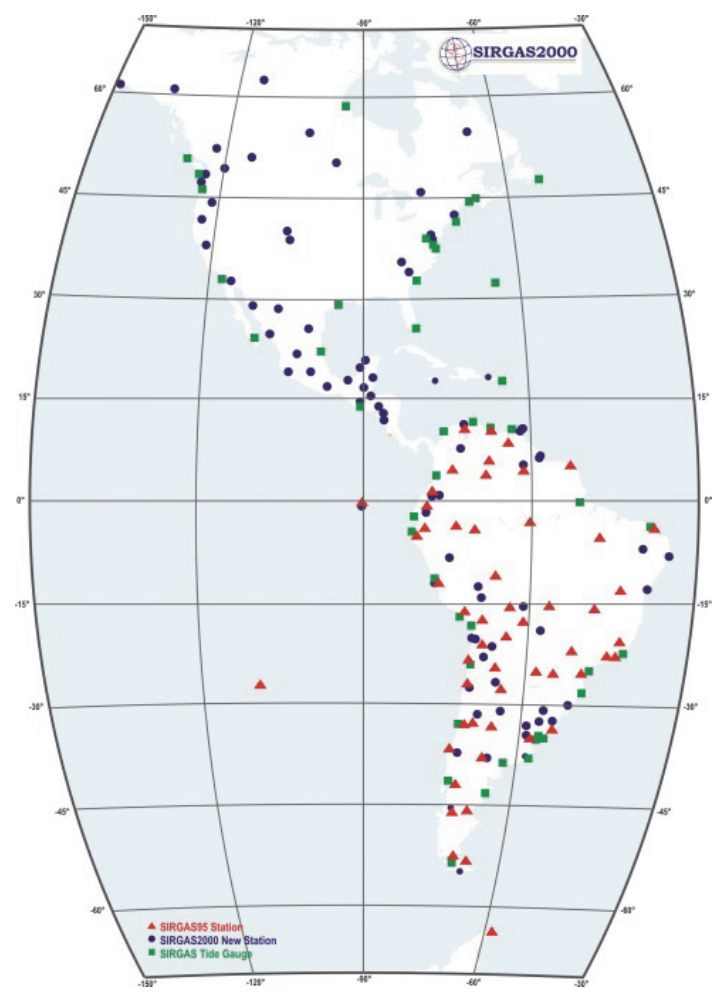

Figura 8. Los 184 sitios que conformaron la red SIRGAS 2000, distribuidos en todo el continente (los triángulos rojos identifican a los puntos SIRGAS95, los cuadrados verdes a los mareógrafos y los círculos azules a los puntos agregados en SIRGAS2000).

\section{De América del Sur a las Américas}

La extensión de la red SIRGAS a América Central, del Norte y al Caribe, y la recomendación promulgada en 2001 por la ONU, a través de su VII Conferencia Cartográfica de las Américas, señalando la adopción de SIRGAS como sistema de referencia oficial en todos los países de las Américas (¡incluyendo a los Estados Unidos y Canadá!), dieron sustento al cambio del significado de las dos últimas siglas de SIRGAS, de "América del Sur" a "las Américas". Ese significado se ha conservado hasta nuestros días, aunque la integración efectiva de los Estados Unidos y Canadá en SIRGAS no fuera más allá de su participación en la campaña de 2000. Los límites geográficos de SIRGAS abarcan hoy a las Américas del Sur y Central, a México y algunas naciones del Caribe (Sánchez y Brunini, 2008; Sánchez y Brunini, 2009; Sánchez et al., 2011; Brunini y Sánchez, 2012; Brunini y Sánchez, 2013). 
Por primera vez en 2004, más precisamente el 9 y 10 de diciembre, SIRGAS se reunió en América del Norte. Aquella reunión, celebrada en las instalaciones del Instituto Nacional de Estadística, Geografía e Informática ${ }^{5}$ (INEGI), en la ciudad de Aguascalientes (México), tuvo el objetivo fundamental de promover la incorporación activa en SIRGAS de México y de los países de América Central. Esa iniciativa habría de fructificar rápidamente con la incorporación de Costa Rica, El Salvador, Guatemala, Honduras, Nicaragua y Panamá, además, por supuesto, del país anfitrión de la reunión.

En la actualidad, SIRGAS orienta sus esfuerzos a la incorporación de los países del Caribe. La próxima Reunión SIRGAS, que tendrá lugar en octubre de 2013, en la ciudad de Panamá, bajo la hospitalidad del Instituto Geográfico Nacional Tommy Guardia, albergará, además de la conmemoración del vigésimo aniversario de SIRGAS, la "School on Reference Systems, Crustal Deformation and Ionosphere Monitoring by GPS in the Caribbean". El uso del idioma inglés y la explicitación "in the Caribbean" en la denominación de la Escuela, anticipa la voluntad de SIRGAS de abrir sus puertas a las naciones del Caribe, que, en su mayoría, no son hispanoparlantes. Es justo mencionar que esa Escuela contará con el auspicio de la IUGG, del IPGH y de la IAG.

\section{De las campañas episódicas a la medición continua}

El concepto sobre el que se apoyaba el mantenimiento de SIRGAS era simple: repitiendo las mediciones en los mismos sitios sería posible determinar los cambios en las posiciones de los puntos; luego, atribuyendo esos cambios a los desplazamientos naturales de los monumentos geodésicos, sería posible calcular la velocidad con que se habían desarrollado esos desplazamientos, mediante el simple cálculo del cociente entre la diferencia de posición y el intervalo transcurrido entre las dos mediciones. Ese concepto, que acepta la aproximación de que los procesos geofísicos que provocan el desplazamiento de los monumentos geodésicos se desarrollan con velocidad constante, permite asignar seis coordenadas a cada punto del marco de referencia: las tres de posición, $X_{0}, Y_{0}, Z_{0}$, válidas para una época de referencia, $T_{0}$, (coincidente con la época media de las mediciones) y las tres de velocidad, $V_{x}, V_{y}, V_{z}$. Con ello, es posible calcular la posición $X$ en cualquier época, $T$, mediante un cómputo tan simple como $X=X_{0}+V x \times\left(T-T_{0}\right)$. Para llevar a la práctica ese concepto (esto es, determinar las velocidades de los puntos SIRGAS95) se remidió en 2000 la red que había sido medida en 1995.

La medición y el cálculo de SIRGAS2000 constituyeron un gran esfuerzo, y también un gran adelanto, por cuanto permitió confirmar la exactitud de SIRGAS95 y evaluar la magnitud de los cambios que habían experimentado las posiciones a lo 
largo de aquellos cinco años. Pero la infraestructura geodésica de la región mejoraba rápidamente con la instalación de estaciones GPS de operación continua (estaciones "CON" en la jerga geodésica). Casi 50 de las 200 estaciones que componían SIRGAS2000 eran CON; y ese número crecía rápidamente (Figura 9), al principio bajo el impulso de la IAG a través de su Servicio Internacional de GNSS (en inglés IGS), y luego por iniciativa de las instituciones de la región, que advertían que las redes de estaciones $\mathrm{CON}$ eran más convenientes que las campañas episódicas para el mantenimiento de sus marcos de referencia (Seemüller y Drewes, 1998a).

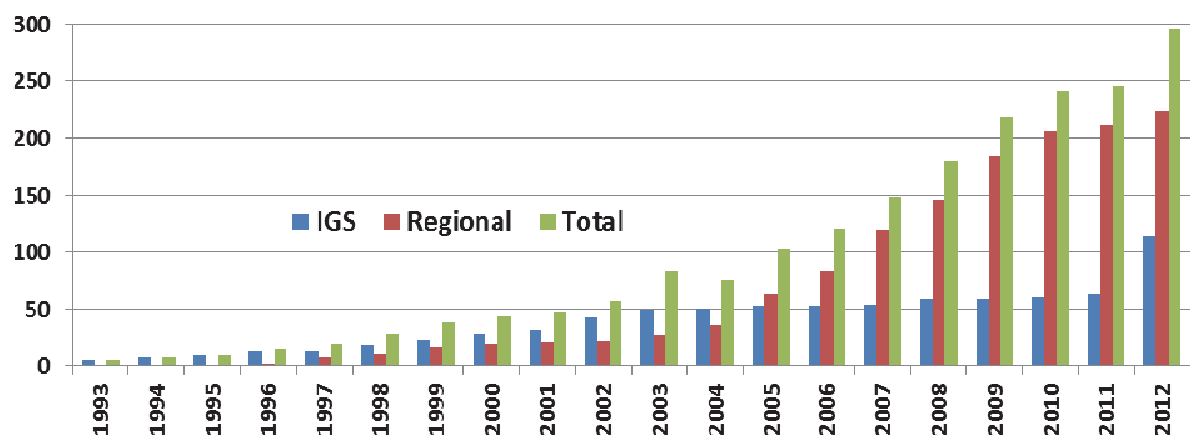

Figura 9. Cantidad de estaciones GNSS de medición continua en la región SIRGAS.

Las estaciones de medición constituyen el cimiento de la infraestructura geodésica actual, pero ningún edificio resulta útil si solo cuenta con sus cimientos: para que las estaciones $\mathrm{CON}$ rindan frutos es necesario generar una compleja estructura de funcionamiento que incluye conectividad para asegurar el transporte de las mediciones, centros de datos para garantizar la perdurabilidad y el acceso a la información y centros de análisis para procesarla y extraer los resultados deseados. Y al igual que las estaciones, esa estructura debe funcionar en modo "CON": ¡las 24 horas de los 365 días del año! La responsabilidad de instalar y operar esa estructura funcional fue asumida por el DGFI en junio de 1996, a través de la creación del Centro de Análisis Asociado al IGS para la Red Regional SIRGAS (en inglés IGS RNAAC SIR; Seemüller y Drewes, 1998b; Seemüller y Drewes, 1999; Seemüller y Drewes, 2000; Seemüller y Drewes, 2002; Seemüller et al., 2002; Seemüller et al., 2004; Seemüller et al., 2008; Seemüller et al., 2010; Seemüller et al., 2012; Seemüller, 2009; Sánchez et al., 2010; Sánchez et al., 2012; Sánchez y Seitz, 2011; Sánchez, 2012).

Ese Centro, que continúa operando, ha mantenido el compromiso de calcular, semana tras semana, diferentes tipos de soluciones que incluyen las mediciones hechas durante toda una semana por todas las estaciones CON de SIRGAS. Esas 
soluciones cumplen tres propósitos fundamentales (Seemüller et al., 2002; Drewes y Heidbach, 2012): i) constituyen la contribución regional de SIRGAS al denominado "Poliedro Global del IGS"; ii) son una "fotografía instantánea" de la red que brinda las mejores coordenadas disponibles en América Latina y el Caribe para apoyar los levantamientos GNSS de alta precisión; y iii) la sucesión de "instantáneas" que se acumula con el paso de los años permite describir con gran precisión los procesos de cambio que afectan a la corteza terrestre en la región SIRGAS (Figura 10). Los autores desean rendir un modesto homenaje a Wolfgang Seemüller (Figura 11), quien desempeño esa tarea con dedicación insuperable hasta su deceso.

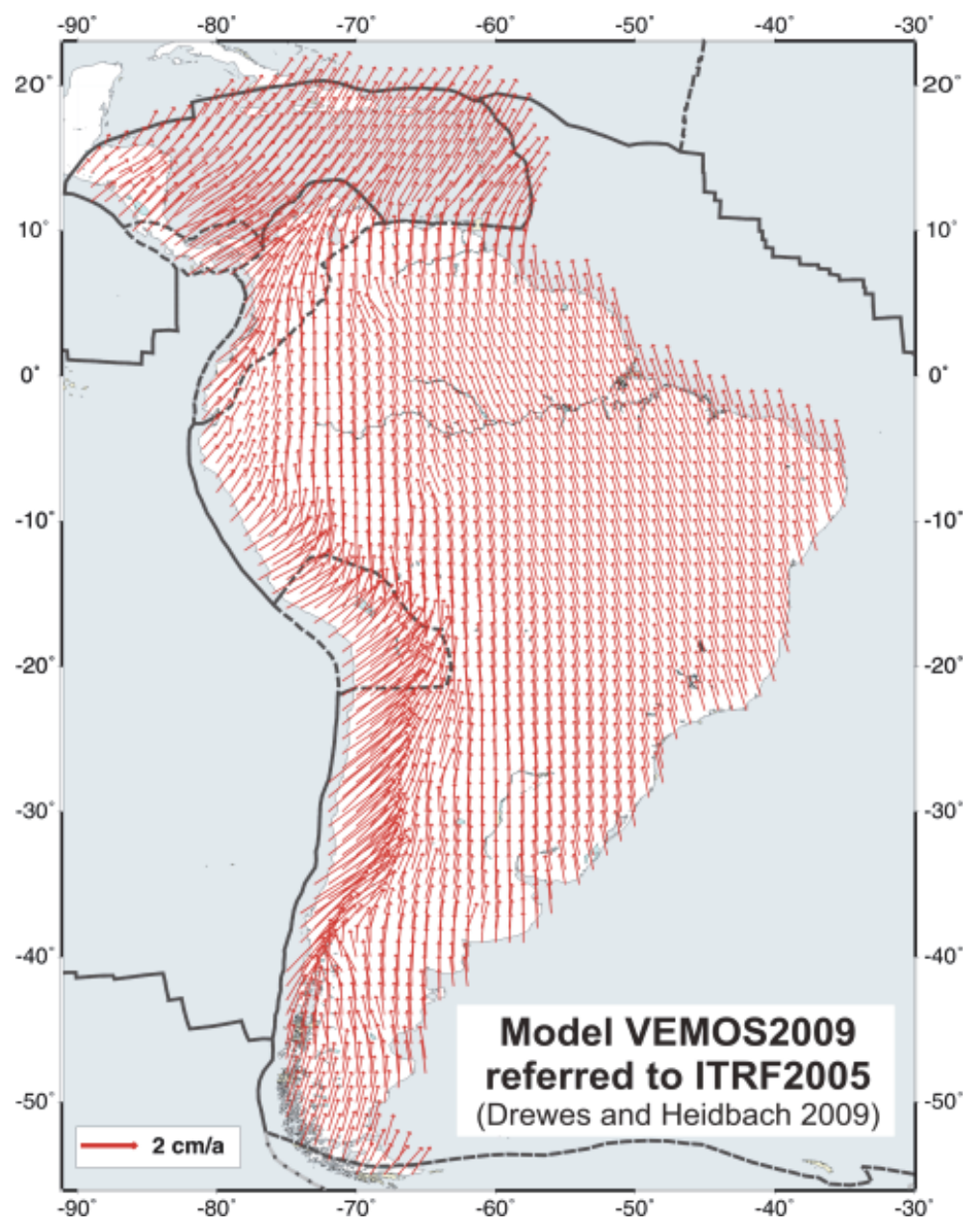

Figura 10. Modelo de Velocidades para SIRGAS (VEMOS2009). 


\section{El crecimiento de SIRGAS CON}

La Figura 9 es elocuente en cuanto a la rapidez con que ha ido creciendo la cantidad de estaciones CON de SIRGAS, desde menos de 50 cuando se instaló el IGS RNAAC SIR, hasta alrededor de 300 en la actualidad. Si apeláramos a la metáfora usada en la sección precedente, deberíamos decir que si crecían los cimientos también debía crecer el edificio, lo que trasladado a la realidad implicaba el crecimiento de la estructura funcional que soportaba el DGFI. Ya en la reunión celebrada en 2004, en Aguascalientes (México), el GT I asignaba la máxima prioridad al fortalecimiento de SIRGAS-CON, incorporando en su agenda las siguientes acciones concretas: i) mejorar el funcionamiento de las estaciones CON que existían en aquel momento; ii) mejorar la transferencia de observaciones a los centros de datos; iii) promover la instalación de nuevas estaciones CON; iv) instalar centros de procesamiento en los países americanos; y v) expandir las capacidades de SIRGAS-CON, posiblemente hacia la generación de productos y servicios en tiempo real y la implantación de un servicio dedicado a monitorizar la atmósfera de la región.

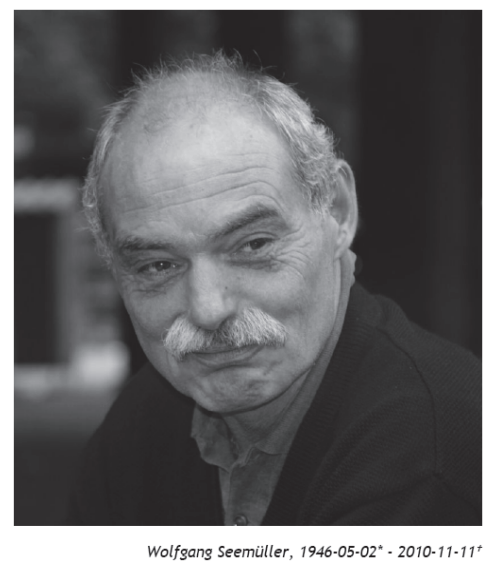

Figura 11. En noviembre de 2010 SIRGAS perdió a uno de sus miembros fundadores y activo impulsor de todas sus iniciativas.

La instalación de centros de procesamiento en los países de América Latina se visualizaba como una necesidad, para distribuir la carga creciente que el procesamiento semanal de SIRGAS-CON imponía sobre el DGFI, pero también como una oportunidad para que el aprendizaje que habían adquirido las instituciones de la región (en gran medida, gracias a la transferencia de conocimientos que ejercía el DGFI), catalizara en nuevas capacidades instaladas en el continente. La Universidad Nacional de La Plata (UNLP) y el IGM (luego IGN), en la Argentina, bajo las responsabilidades de Paula Natali y Sergio Cimbaro, el IBGE, en Brasil, bajo la de 
Sonia Costa, y el INEGI, en México, bajo la de Guido González, habían dado ya los primeros pasos, instalando centros de procesamiento experimentales que sobrellevaban la tarea de calcular soluciones semanales de entre 15 y 35 estaciones SIRGAS-CON. Pero convertir aquel experimento en una estructura funcional que soportara el paso del tiempo y el peso de la responsabilidad de generar las soluciones oficiales de SIRGAS, sería una tarea que insumiría gran parte de los esfuerzos por venir: el tema fue motor de extensos debates en las reuniones que se celebrarían el 17 y 18 de noviembre de 2005, en Caracas (Venezuela), bajo la hospitalidad de la Sección Nacional venezolana del IPGH; el 27 y 28 de noviembre de 2006, en Heredia (Costa Rica), bajo la hospitalidad de la Universidad Nacional (UNA); y el 7 y 8 de junio de 2007, en Bogotá (Colombia), bajo la hospitalidad del IGAC.

El hecho fundacional de aquella empresa, cuya meta más ambiciosa permanece aún abierta y es la instalación de al menos un centro de procesamiento en cada país integrante de SIRGAS, se concretó con el Primer Taller del GT I, celebrado en Río de Janeiro (Brasil), entre el 16 y el 18 de agosto de 2006 bajo la hospitalidad del IBGE. Hubo que trabajar muy intensamente para que aquellas tres jornadas alcanzaran para definir los lineamientos fundamentales del proyecto "Centros de Análisis para SIRGAS”, los cuales: i) ratificaban el trabajo de los cuatro centros de procesamiento experimentales antes mencionados, a los que se había sumado uno instalado en IGAC, Colombia, bajo la responsabilidad de William Martínez; ii) asignaban a cada centro la responsabilidad de procesar una subred de SIRGASCON y fijaban los estándares de procesamiento; iii) establecían un centro de combinación en DGFI, bajo la responsabilidad de Wolfgang Seemüller, y otros dos experimentales, en IBGE y UNLP bajo las responsabilidades de Sonia Costa y Paula Natali; iv) imponían a los centros de procesamiento la obligación de enviar sus soluciones semanales a los centros de combinación, dentro de las tres semanas siguientes a la de medición; e v) imponían a los últimos la obligación de comparar las diferentes soluciones y combinarlas en la solución definitiva dentro del plazo de una semana y llevar una estadística sobre el desempeño de los diferentes centros.

El siguiente acto relevante ocurrió durante el Segundo Taller del GT I, celebrado el 26 y 27 de mayo de 2008, en Montevideo (Uruguay), bajo la hospitalidad del Servicio Geográfico Militar, institución que también hospedó la Reunión SIRGAS que se celebró inmediatamente después, entre el 28 y el 30 del mismo mes. Durante aquel acontecimiento, que coincidía con la conmemoración de los primeros 100 años de la Geodesia en Uruguay (conmemoración que ya se había dado o estaba por darse en la mayoría de los jóvenes países latinoamericanos), la comunidad SIRGAS en pleno evaluó los casi dos años de trabajo de los centros experimentales de procesamiento y combinación. Los resultados fueron extraordinariamente alentadores: los de la Universidad Nacional de Cuyo (UNC), de la Argentina, que bajo la responsabilidad de Virginia Mackern continuaba la tarea que se había iniciado en la 
UNLP, de IBGE y de IGAC, cumplían los estándares de calidad, puntualidad y continuidad para dejar de ser experimentales y convertirse en oficiales; el desempeño del IGM argentino y el INEGI mexicano garantizaba una transición inminente del estatus experimental al oficial; y los centros de combinación experimentales del DGFI y el IBGE también satisfacían los estándares impuestos para convertirse en oficiales. ¡El edificio contaba ya con su planta baja!

\section{Un centro de procesamiento SIRGAS en cada país}

El proceso iniciado por SIRGAS hace casi diez años para dotar a todos los países de América Latina y el Caribe de por lo menos un centro de procesamiento, continúa vigoroso. En la actualidad, SIRGAS-CON cuenta con más de 300 estaciones (Figura 12) y las previsiones denuncian que ese número continuará creciendo a ritmo sostenido. Su procesamiento es llevado a cabo, en forma permanente y con una cadencia semanal, por 10 centros de procesamiento oficiales, un centro de procesamiento experimental (candidato a convertirse en oficial) y dos centros de Combinación oficiales (Tabla 1).

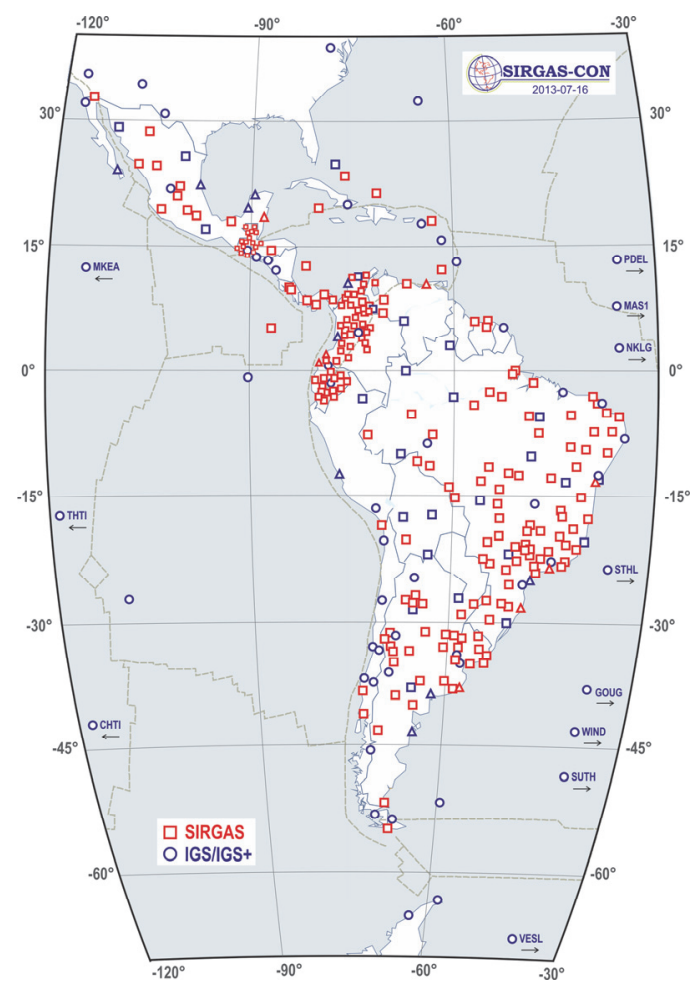

Figura 12. Red SIRGAS CON (estado al 17 de julio de 2013). 
Tabla 1

Centros de Procesamiento y Combinación de SIRGAS (estado al 17 de julio de 2013)

\begin{tabular}{|c|c|}
\hline Institución & Responsables \\
\hline \multicolumn{2}{|l|}{ Centros de Procesamiento oficiales } \\
\hline $\begin{array}{l}\text { Deutsches Geodätisches } \\
\text { Forschungsinstitut, Alemania } \\
\text { (responsable del IGS RNAAC SIR) }\end{array}$ & Laura Sánchez \\
\hline Instituto Geográfico Agustín Codazzi, & Orlando López, William Martínez, Francisco \\
\hline Colombia & Mora, Oscar Suárez y Santiago Venegas \\
\hline Instituto Brasileiro de Geografia e & Marco de Almeida Lima, Newton Junior y \\
\hline Estatistica, Brasil & Claudia Santos Silva \\
\hline Instituto Geográfico Militar, Chile & $\begin{array}{l}\text { Cristian Mardones Castro, Héctor Parra Bravo, } \\
\text { Víctor Piña Acuña y Sergio Rozas Bornes }\end{array}$ \\
\hline Instituto Geográfico Militar, Ecuador & $\begin{array}{l}\text { Marco Amores, Alberto Chávez y David } \\
\text { Cisneros }\end{array}$ \\
\hline Instituto Geográfico Nacional, Argentina & $\begin{array}{l}\text { Sergio Cimbaro, Agustín Raffo y Diego } \\
\text { Piñón }\end{array}$ \\
\hline Instituto Nacional de Estadística y & Luís Becerrill, Guillermo Gasca y Guido \\
\hline Geografía, México & González \\
\hline Servicio Geográfico Militar, Uruguay & $\begin{array}{l}\text { Gonzalo Campo, Mathias Manisera Urciuoli, } \\
\text { José Pampillón, Juan Perlas, Pedro Sandoval, } \\
\text { Norbertino Suárez y Julio Teixeira }\end{array}$ \\
\hline Universidad del Zulia, Venezuela & Víctor Cioce y Dhanniela Espinoza \\
\hline Universidad Nacional de Cuyo, Argentina & $\begin{array}{l}\text { Andrea Calori, Fernanda Camisay, Virginia } \\
\text { Mackern, Laura Mateo y Ana Robín }\end{array}$ \\
\hline \multicolumn{2}{|l|}{ Centros de Procesamiento experimentales } \\
\hline Universidad Nacional, Costa Rica & $\begin{array}{l}\text { Sara Bastos Gutiérrez, Jorge Moya Zamora, } \\
\text { María José Rivas Guzmán y Francisco } \\
\text { Valverde Calderón }\end{array}$ \\
\hline \multicolumn{2}{|l|}{ Centros de Combinación Oficiales } \\
\hline $\begin{array}{l}\text { Instituto Brasileiro de Geografia e } \\
\text { Estatistica, Brasil }\end{array}$ & Alberto da Silva \\
\hline $\begin{array}{l}\text { Deutsches Geodätisches } \\
\text { Forschungsinstitut, Alemania }\end{array}$ & Laura Sánchez \\
\hline
\end{tabular}

Esta estructura funcional permite el procesamiento desagregado de SIRGASCON: el DGFI procesa una subred de extensión continental y los otros centros de procesamiento oficiales procesan subredes que se extienden sobre el país al que pertenece el centro y su región aledaña. Todas las estaciones SIRGAS-CON son calculadas en forma independiente por al menos tres centros de procesamiento y todas las soluciones resultantes son combinadas por el IBGE, como centro primario de combinación, y por el DGFI, como centro de resguardo y control. De este modo, las soluciones semanales que SIRGAS ofrece a la comunidad, en forma libre y gratuita, han superado controles redundantes que garantizan la mayor confiabilidad en el cumplimiento de los estándares geodésicos más exigentes en el ámbito internacional (Sánchez et al., 2008; Sánchez et al., 2012; Costa et al., 2012). 


\section{Creación permanente de capacidades}

La instalación de centros de procesamiento en los países de América Latina y el Caribe da cuenta del compromiso de SIRGAS con la creación permanente de capacidades. Ese compromiso se sostiene con una variedad de acciones complementarias que abarcan acuerdos institucionales, cursos, talleres y escuelas, articuladas por una política que se evalúa y ajusta anualmente en las reuniones del Consejo Directivo de SIRGAS.

Ningún centro de procesamiento, experimental u oficial, es reconocido por SIRGAS de no mediar un compromiso institucional debidamente documentado de sostener su funcionamiento. Entre los acuerdos institucionales que soportan la instalación de centros de procesamiento es necesario resaltar el que han suscrito varias instituciones con el DGFI, y a través de éste, con la Universidad de Berna (Suiza), para acceder, de forma muy conveniente, al programa de procesamiento Bernese, y a la capacitación para utilizarlo dentro de los objetivos de SIRGAS. Esa capacitación ha incluido cursos in situ, que en su mayoría fueron impartidos por la actual vicepresidente de SIRGAS, Laura Sánchez, de DGFI (Alemania); y en menos casos por la actual presidente del Grupo de Trabajo I, Virginia Mackern, de la UNC (Argentina).

La actividad paradigmática en materia de creación de capacidades ha sido la "Escuela SIRGAS/IAG/IPGH", que se ha desarrollado con frecuencia anual desde 2009, convocando en cada edición a más de un centenar de asistentes provenientes de la mayoría de los países de la región (Figura 13). Las tres primeras se dedicaron a los "Sistemas de Referencia" y tuvieron lugar en Bogotá (Colombia), entre el 13 y el 17 de junio de 2009, bajo la hospitalidad del IGAC; en Lima (Perú), entre el 8 y el 10 de noviembre de 2010, bajo la hospitalidad del IGN; y en Heredia (Costa Rica), entre el 3 y el 5 de agosto de 2011, bajo la hospitalidad de la UNA. Las clases fueron impartidas por Hermann Drewes y Laura Sánchez (DGFI, Alemania), William Martínez (IGAC, Colombia), Virginia Mackern (UNC, Argentina) y Claudio Brunini (UNLP, Argentina).

La cuarta y última de las realizadas a la fecha, abordó la temática del "Posicionamiento GNSS en Tiempo Real" y se desarrolló en Concepción (Chile), entre el 24 y 26 de octubre de 2012, bajo la hospitalidad de la Universidad de Concepción (UC) y del IGM de Chile. En este caso las clases fueron impartidas por expertos de la Agencia Federal Alemana para Cartografía y Geodesia (BKG) con la cooperación de Roberto Pérez Rodino (Universidad de la República —UdeLaR—, Uruguay), Gustavo Noguera (Universidad Nacional de Rosario - UNR - , Argentina) y Melvin Hoyer (LUZ, Venezuela).

La otra actividad relevante orientada a la creación de capacidades han sido los talleres ya mencionados, desarrollados por el GT I en dos ocasiones (Río de Janeiro, Brasil, del 16 al 18 de agosto de 2006 y Montevideo, Uruguay, del 26 al 27 de mayo de 2008) y por el GT III en otras dos ocasiones (Santiago, Chile, del 10 al 14 de agosto de 1998 y Río de Janeiro, Brasil, del 3 al 6 de diciembre de 2012). 


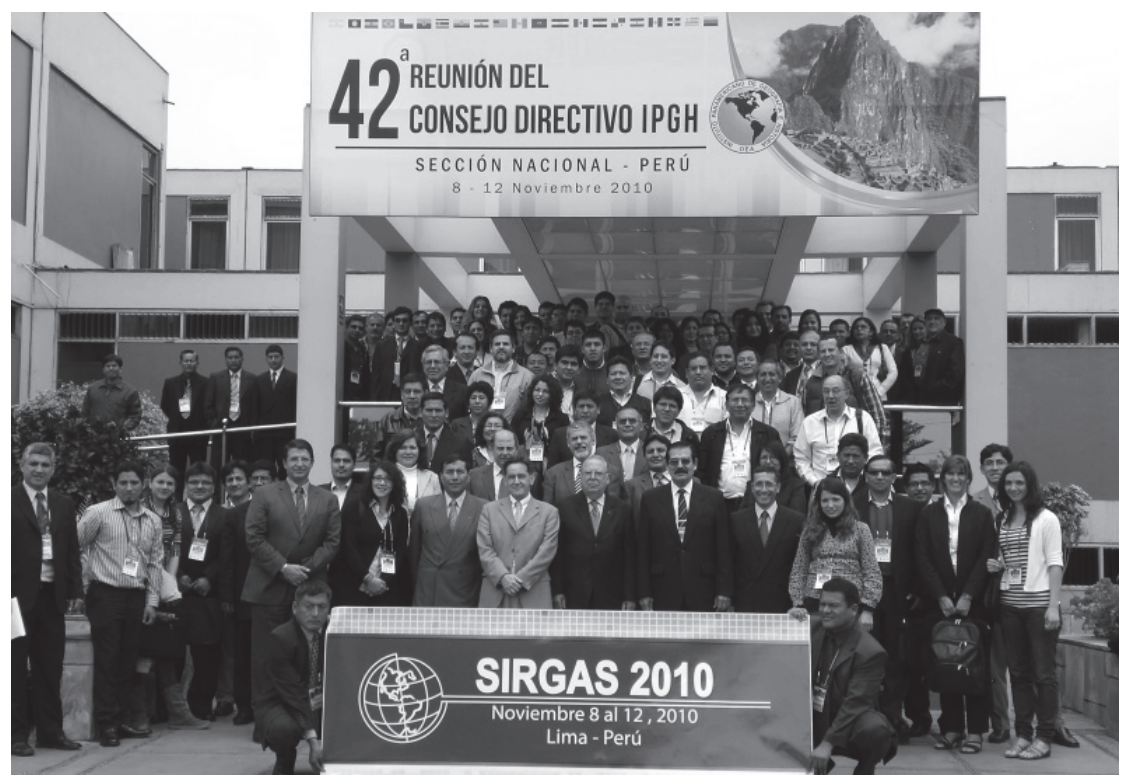

Figura 13. Fotografía grupal de los asistentes a las Escuela SIRGAS/IAG/IPGH de 2010.

Las reuniones que se han llevado a cabo desde 1993 con regularidad casi anual (Tabla 2), constituyen el principal punto de encuentro (presencial, dado que la Internet se ha convertido en un medio de encuentro virtual usado con mucha frecuencia por SIRGAS) de todas las componentes de la Organización: el Consejo Directivo, que evalúa los avances logrados en los diferentes campos de SIRGAS y define las directrices para la etapa venidera; el Comité Ejecutivo, cuya responsabilidad es velar por el cumplimiento de aquellas directrices; el Consejo Científico, que los asesora; y los Grupos de Trabajo, que son los artífices primarios de los logros de SIRGAS.

La modalidad de las reuniones cambió en 2008: a partir de ese año SIRGAS abrió a toda la comunidad las reuniones que originalmente convocaban solamente a los integrantes de sus cuerpos orgánicos. Bajo la denominación de "Reunión SIRGAS..." (los puntos suspensivos corresponden al año), se han convocado encuentros con más de un centenar de asistentes y varias decenas de exposiciones científicas que superan las revisiones habituales en los simposios de la especialidad. Nada dimensiona mejor la magnitud del crecimiento que la fotografía grupal de la Reunión SIRGAS, 2012, tomada durante la visita que los asistentes realizaron al observatorio geodésico fundamental TIGO, instalado en la ciudad chilena de Concepción (Figura 14). Los apoyos del IPGH y la IAG han sido fundamentales para permitir la transformación que ha convertido a las reuniones SIRGAS en el mayor foro geodésico de América Latina. 
Tabla 2

Reuniones sostenidas por las diferentes componentes de SIRGAS

\begin{tabular}{|c|c|c|}
\hline Reunión & Lugar y fecha & Institución anfitriona \\
\hline GT II & Bogotá, Colombia, 20-22 abril de 1994 & IGAC \\
\hline GT I y GT II & $\begin{array}{l}\text { La Plata, Argentina, 24-28 de octubre de } \\
1994\end{array}$ & $\begin{array}{l}\text { Asociación Argentina } \\
\text { de Geofísicos y } \\
\text { Geodestas }\end{array}$ \\
\hline GT I y GT II & $\begin{array}{l}\text { Santiago de Chile, Chile, 5-9 de agosto de } \\
1996\end{array}$ & IGM \\
\hline $\begin{array}{l}\text { Comité } \\
\text { Ejecutivo }\end{array}$ & $\begin{array}{l}\text { Isla Margarita, Venezuela, 8-11 de abril de } \\
1997\end{array}$ & LUZ e IGM \\
\hline $\begin{array}{l}\text { Comité } \\
\text { Ejecutivo }\end{array}$ & $\begin{array}{l}\text { Río de Janeiro, Brasil, 3-9 de septiembre de } \\
1997\end{array}$ & IAG \\
\hline GT III & $\begin{array}{l}\text { Santiago de Chile, Chile, 11-13 de agosto de } \\
1998\end{array}$ & IGM \\
\hline GT I y GT III & $\begin{array}{l}\text { Birmingham, Inglaterra, 27-28 de julio de } \\
1999\end{array}$ & IUGG \\
\hline $\begin{array}{l}\text { Comité } \\
\text { Ejecutivo }\end{array}$ & $\begin{array}{l}\text { Cartagena, Colombia, } 20-23 \text { de febrero de } \\
2001\end{array}$ & IAG \\
\hline GT I y GT III & $\begin{array}{l}\text { Budapest, Hungría, 2-7 de septiembre de } \\
2001\end{array}$ & IAG \\
\hline $\begin{array}{l}\text { Comité } \\
\text { Ejecutivo }\end{array}$ & $\begin{array}{l}\text { Santiago de Chile, Chile, } 21-25 \text { de octubre de } \\
2002\end{array}$ & IGM \\
\hline $\begin{array}{l}\text { Comité } \\
\text { Ejecutivo }\end{array}$ & $\begin{array}{l}\text { Aguascalientes, México, 9-10 de diciembre } \\
\text { de } 2004\end{array}$ & INEGI \\
\hline Comité & Caracas, Venezuela, 17-18 de noviembre de & Sección Nacional del \\
\hline Ejecutivo & 2005 & IPGH \\
\hline GT I y GT II & $\begin{array}{l}\text { Heredia, Costa Rica, 27-28 de noviembre de } \\
2006\end{array}$ & UNA \\
\hline $\begin{array}{l}\text { Comité } \\
\text { Ejecutivo }\end{array}$ & Bogotá, Colombia, 7-8 de junio de 2007 & IGAC \\
\hline SIRGAS2008 & $\begin{array}{l}\text { Montevideo, Uruguay, 28-30 de mayo de } \\
2008\end{array}$ & SGM \\
\hline SIRGAS2009 & $\begin{array}{l}\text { Buenos Aires, Argentina, } 31 \text { de agosto- } 4 \text { de } \\
\text { septiembre de } 2009\end{array}$ & IAG \\
\hline SIRGAS2010 & Lima, Perú, 11-12 de noviembre de 2010 & IGN \\
\hline SIRGAS2011 & Heredia, Costa Rica, 8-10 de agosto de 2011 & UNA \\
\hline SIRGAS2012 & Concepción, Chile, 29-31 de octubre de 2012 & UC e IGM \\
\hline
\end{tabular}

\section{SIRGAS de cara al futuro}

El encuadre institucional de SIRGAS se sustenta en dos paradigmas complementarios: el establecido por la IAG para su "Sistema Geodésico de Observación Global" (en inglés GGOS) y el definido por el IPGH en su "Agenda Panamericana 20102020". 


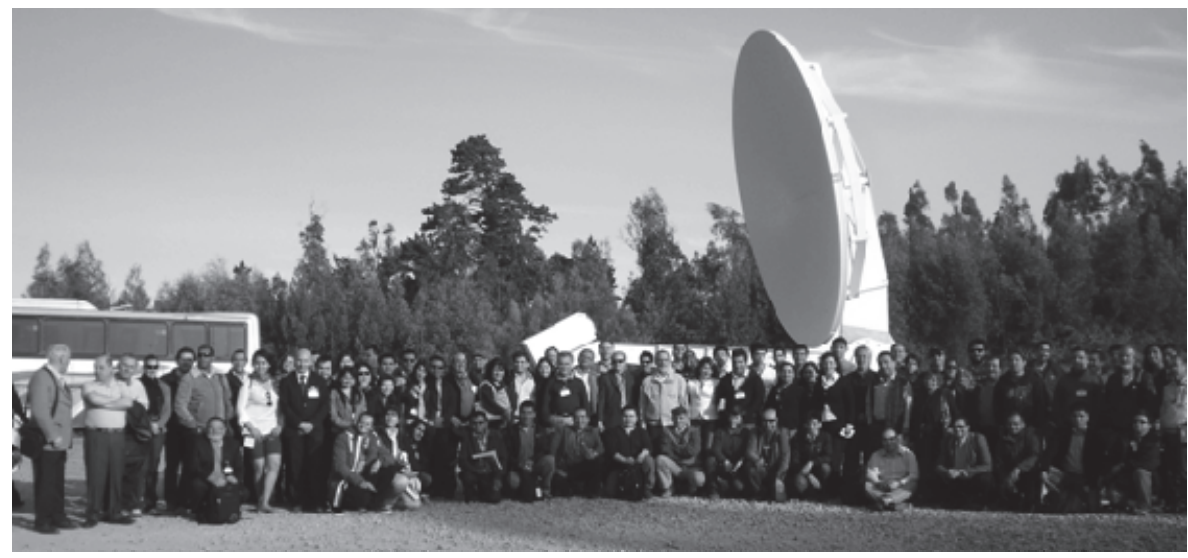

Figura 14. Fotografía grupal de la Reunión SIRGAS2012, tomada durante la visita al Observatorio Geodésico Fundamental TIGO.

El primero de esos paradigmas expresa la vanguardia científica y tecnológica internacional en el campo de la Geodesia, en tanto que el segundo pone el acento en el uso de los conocimientos y las tecnologías de base geodésica para mejorar el ordenamiento territorial, mitigar el efecto de los desastres y abordar los procesos de cambio climático global que afectan a las Américas. Dentro de ese contexto, SIRGAS se conceptualiza a sí mismo como un puente que, transitado en una dirección, promueve la participación de la región en el escenario geodésico internacional, y transitado en la dirección opuesta, asegura que los conocimientos científicos de avanzada se transformen en soluciones concretas para los problemas de base geodésica que plantea la región.

La columna vertebral de la Agenda Panamericana 2010-2020 es la "Infraestructura de Datos espaciales de las Américas" (IDEA), a cuyo desarrollo el IPGH contribuye brindando soporte a tres organizaciones panamericanas: CPIDEA, GeoSUR y SIRGAS. El 15 de noviembre de 2012, durante la 44a Reunión del Consejo Directivo del IPGH celebrada en Buenos Aires (Argentina), esas tres organizaciones suscribieron junto al IPGH el "Plan de Acción Conjunto 2013-2015 para acelerar el desarrollo de la IDE de las Américas”. Resulta difícil abstenerse a la tentación de usar una imagen de capas apiladas si se deseara crear un logotipo que identifique a una IDE. Pero esa imagen, que sintetiza tan bien el concepto fundamental de la IDE, no dice nada sobre cómo se logran alinear las capas para que se superpongan unas sobre las otras. Suele decirse que eso se logra con la georreferenciación, pero aunque necesaria, esa condición es insuficiente para garantizar el alineamiento de las capas. Es imprescindible, además, que la georreferenciación de todas las capas se haga con respecto a un único marco de referencia, que constituye la capa funda- 
mental de la IDE. Ese marco de referencia para América Latina y el Caribe lo proporciona SIRGAS.

Además de ese insumo básico, SIRGAS contribuye al desarrollo de la IDEA mejorando las capacidades de los productores y usuarios de información espacial a través de los cursos, talleres, escuelas y reuniones que se han descrito en la sección precedente; mediante la elaboración de estándares, especificaciones y guías de trabajo aplicables a la región; y coordinando una infraestructura de medición, almacenamiento y diseminación de datos de la red SIRGAS-CON. En relación con este último ítem, sobresalen los proyectos:

- "SIRGAS en Tiempo Real", orientado a mejorar la capacidad de de SIRGASCON para la georreferenciación en tiempo real (Figura 15a); fue instalado en 2008 bajo la coordinación de Melvin Hoyer (LUZ, Venezuela) y luego la de Roberto Pérez Rodino (UdeLaR, Uruguay) y Gustavo Noguera (UNR, Argentina).

- "SIRGAS GLONASS", orientado a incorporar las mediciones de los satélites GLONASS en el procesamiento de SIRGAS-CON (Figura 15b), fue instalado en 2011 bajo la coordinación de Víctor Cioce (LUZ, Venezuela) y Ana María Robín (UNC, Argentina).
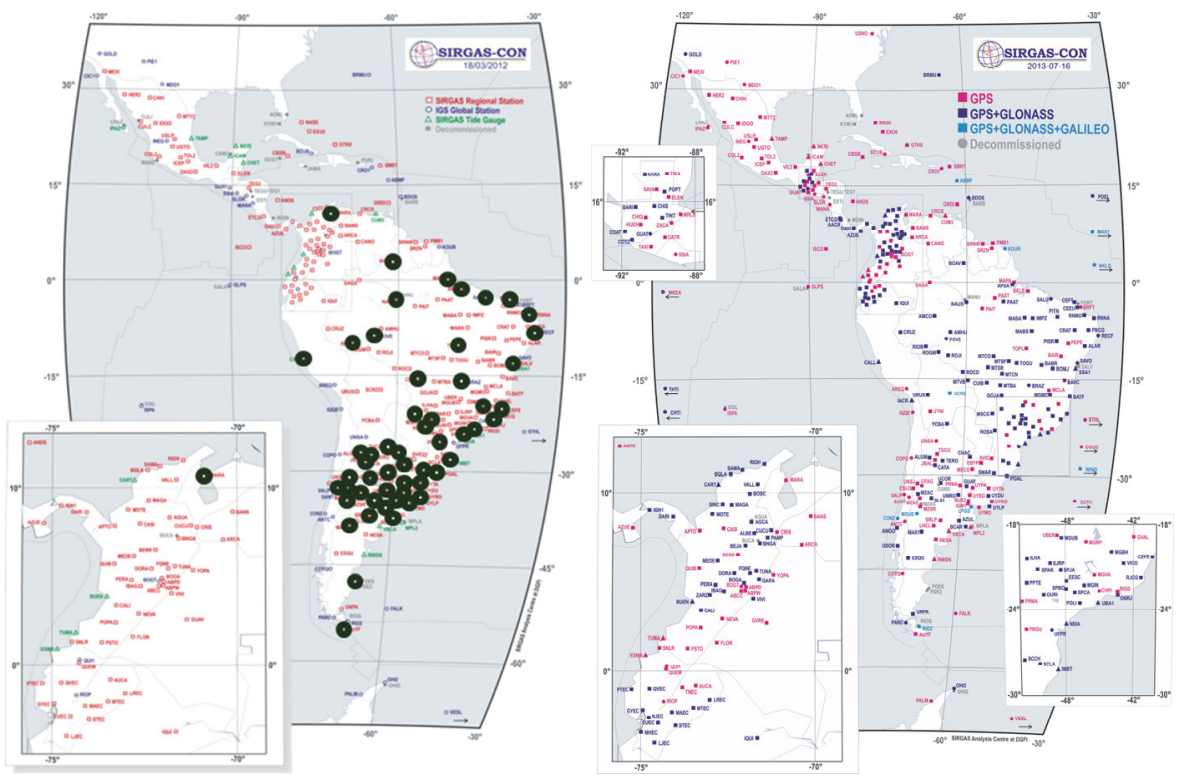

Figura 15. Distribución actual de las estaciones SIRGAS-CON a) con capacidad de transmisión de datos tiempo real (izquierda); y b) que capturan, además de GPS, señales GLONASS o Galileo (derecha). 
LPIM REGIONAL IONOSPHERE DAY:045, YEAR: 2012
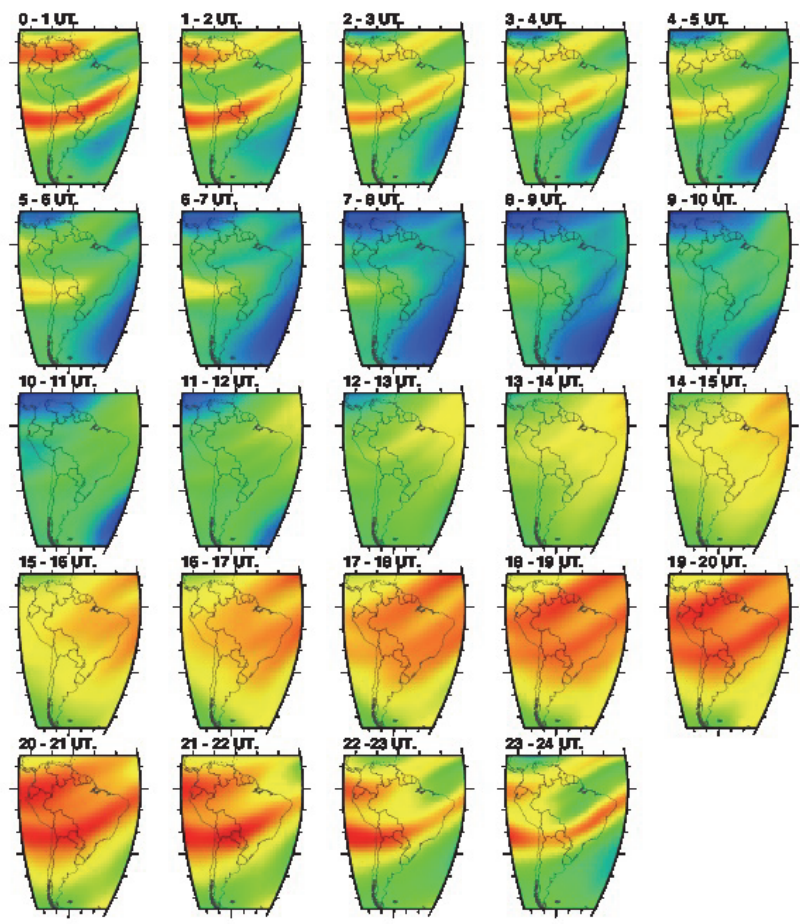

$14-15$ vr.
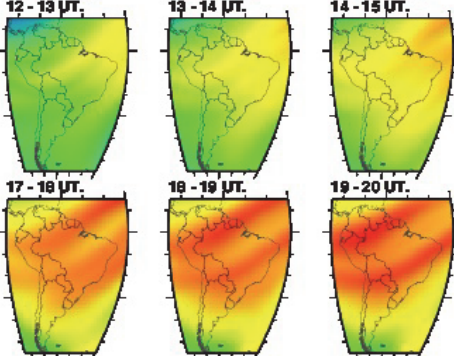

$22-204$.
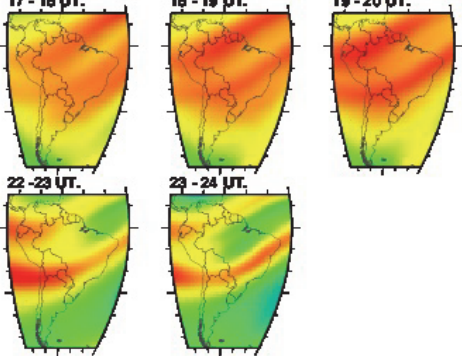

$0 \quad 1020 \quad 30 \quad 40 \quad 50 \quad 6070 \quad 80$

[TECU]

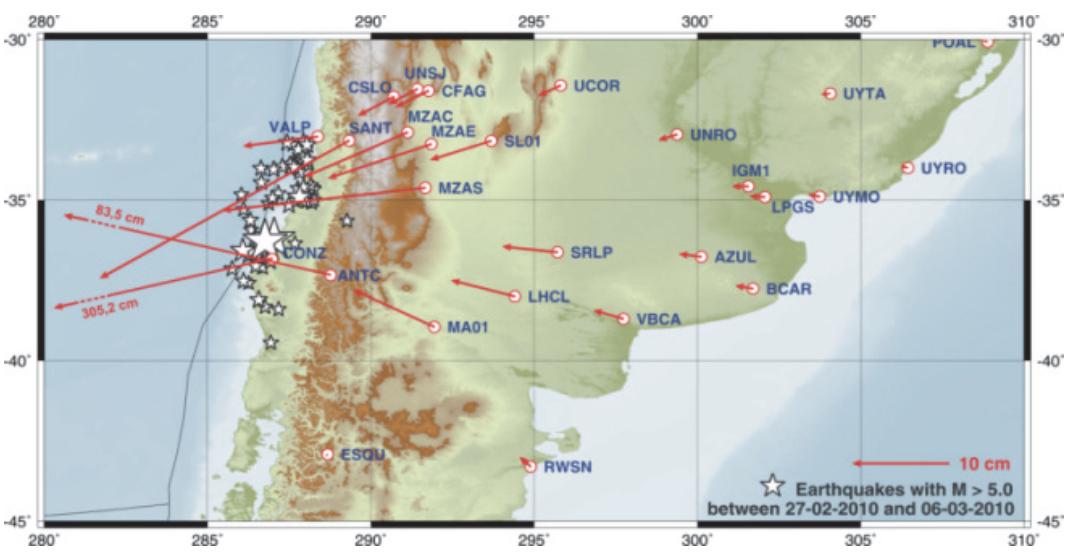

Figura 16a Secuencia de mapas que describen la variación del contenido total de electrones en la ionosfera a lo largo de un día; y b) desplazamiento causados en las estaciones SIRGAS-CON por el terremoto de El Maule (Chile, febrero de 2010). 
El paradigma actual de la IAG impone la materialización de un marco de referencia que soporte todas las demandas de las IDE - desde el mapeo de grandes territorios despoblados hasta la cartografía de las más densas urbanizaciones-, y sirva también para el posicionamiento milimétrico requerido para estudiar la geodinámica y el cambio global (Drewes, 2012; Brunini et al., 2012; Sánchez et al., 2013). Los centros de procesamiento y combinación de SIRGAS le entregan a la comunidad un marco de referencia acorde a ese paradigma. Pero el compromiso de SIRGAS con la problemática del cambio global incluye también el desarrollo de varios proyectos que, aprovechando las mediciones SIRGAS-CON, producen información que contribuyen a comprender la actividad tectónica, la variabilidad de la atmósfera, el aporte de diferentes procesos geofísicos al ciclo del agua, las variaciones del nivel del mar en ambas costas del continente americano, etc. Dichas investigaciones se enmarcan en los proyectos:

- "Estudios Atmosféricos basados en la infraestructura de SIRGAS", que contiene dos componentes: una dedicada al contenido electrónico en la ionosfera (Figura 16a, Brunini et al., 2008), establecido en 2007 bajo la coordinación de Mauricio Gende (UNLP, Argentina); y otra dedicada al vapor de agua en la atmósfera neutra, que incluye contribuciones realizadas por grupos de Argentina, Colombia, Ecuador y Venezuela (e.g. Cioce et al., 2010);

- "Movimientos No Lineales", cuya meta es perfeccionar el mantenimiento del marco de referencia SIRGAS mediante el modelado de los procesos geofísicos que ocasionan cambios en las posiciones de las estaciones que no son lineales en el tiempo (e.g. terremotos, Figura 16b), variaciones estacionales inducidas por masas hidrológicas o atmosféricas, etc.; Kaniuth et al., 2002b; Báez et al., 2007; Sánchez et al., 2010; Cruz Ramos y Sánchez, 2012; Bruyninx et al., 2012); este proyecto fue instalado en 2011 y es coordinado por Juan Báez (UC, Chile) y Sergio Cimbaro (IGN, Argentina).

\section{La estructura orgánica de SIRGAS}

A lo largo de la primera década, la vida de SIRGAS estuvo regulada por acuerdos que no habían sido formalizados, más allá de las constancias escritas que dejaban los boletines que redactaba y distribuía el Presidente del Comité del Proyecto. La estructura funcional, que había sido sugerida por Hermann Drewes en la reunión fundacional de 1993 (Figura 17), produjo resultados admirables a lo largo de toda una década. Hacia el inicio de la segunda, SIRGAS había crecido lo suficiente para requerir una estructura funcional más compleja y, de su mano, una formalización más rigurosa de sus pautas de funcionamiento. Ello condujo a la elaboración de un Estatuto que fue discutido y aprobado en la Reunión de 2002, en Santiago de Chile. 

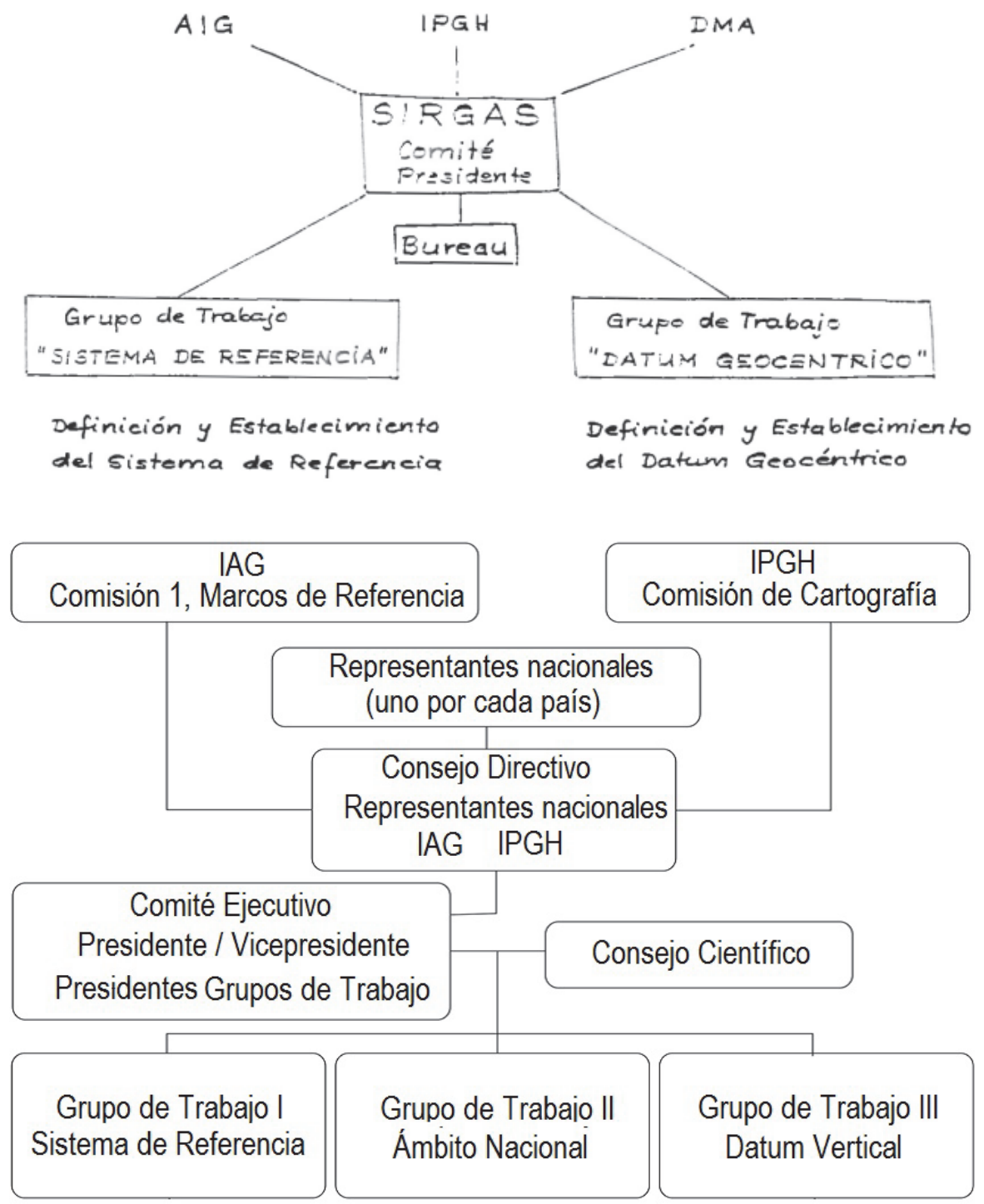

Figura 17. Arriba: reproducción de la lámina utilizada por H. Drewes en la Reunión de 1993, para proponer la estructura funcional de SIRGAS que perduraría por más de una década; debajo: estructura funcional actual de SIRGAS. 
Con modificaciones formales que se aprobaron en 2011 — en la Reunión que se realizara entre el 8 y el 10 de agosto de ese año, en Heredia (Costa Rica)-, el Estatuto consagra el carácter democrático de SIRGAS, confiriendo la máxima autoridad a un Consejo Directivo (CD) formado por un representante de cada país miembro (19 en la actualidad) y uno de cada entidad patrocinadora (la IAG y el IPGH). Todos los estados americanos y del Caribe pueden ser miembros de SIRGAS y designar a sus representantes a través de sus organismos competentes. Ese Consejo fija las políticas de fondo mediante un mecanismo que privilegia el debate y la búsqueda de consensos y asigna un voto a cada uno de sus integrantes. Subordinado al CD existe un Comité Ejecutivo (CE), sobre el que recae la responsabilidad de llevar a la práctica las políticas decididas por el CD (Figura 9). Cuenta para ello con el asesoramiento de un Consejo Científico (CC) integrado por especialistas reconocidos en el ámbito internacional. El CE está integrado por el presidente y vicepresidente de SIRGAS y por los presidentes de los GT (tres en la actualidad). El presidente y el vicepresidente de SIRGAS son elegidos por el CD y renovados cada cuatro años.

Los Grupos de Trabajo están abiertos a toda persona calificada que desee contribuir al cumplimiento de los objetivos de SIRGAS y asuma el compromiso de honrar el significado de la sigla "T", i.e. trabajo. Sus presidentes son elegidos por el presidente de SIRGAS con el aval del CD. El Estatuto de SIRGAS consagra la ausencia de lucro en los objetivos de la organización, garantiza el acceso irrestricto a sus conocimientos, productos y servicios y asegura el respeto a las políticas de datos que cada estado establece en forma independiente.

Inmediatamente aprobado el Estatuto, se ratificó a Luiz Paulo Souto Fortes, del IBGE, en la presidencia de SIRGAS y se eligió al primer vicepresidente, recayendo la responsabilidad sobre Eduardo Lauría, del IGM argentino. ${ }^{6} \mathrm{Al}$ expirar sus mandatos, una nueva elección concretada en la Reunión del 7 y 8 de junio de 2007, en Bogotá (Colombia), bajo la hospitalidad del IGAC, asignó esas responsabilidades a los autores del presente artículo, quienes resultaron reelectos en la reunión del 8 al 10 de agosto de 2011, en Heredia (Costa Rica). La Tabla 3 presenta la lista de los diferentes integrantes del CE de SIRGAS.

\section{SIRGAS en la Web}

El portal web de SIRGAS <www.sirgas.org> ha acompañado el crecimiento del proyecto y ha evolucionado al ritmo que crecía la importancia de la Internet como medio de integración regional y global y como fuente de información y conocimientos. En la actualidad contiene la información más completa, detallada y precisa que pueda hallarse sobre SIRGAS y es la principal boca de acceso a los productos que SIRGAS le entrega a la comunidad. Su actualización es permanente y no sería 
exagerado caracterizarla con la terminología tan en boga dentro de la comunidad geodésica de "en tiempo real".

Tabla 3

Miembros actuales y pasados del Comité Ejecutivo de SIRGAS

\begin{tabular}{|c|c|c|}
\hline Periodo & Responsable & Institución \\
\hline \multicolumn{3}{|c|}{ Presidentes SIRGAS } \\
\hline $\begin{array}{l}1993-2002 \\
2002-2007\end{array}$ & Luiz Paulo Souto Fortes & $\begin{array}{l}\text { Instituto Brasileiro de Geografia e Estatística, } \\
\text { Brasil }\end{array}$ \\
\hline $\begin{array}{l}2007-2011 \\
2011-2015\end{array}$ & Claudio Brunini & Universidad Nacional de La Plata, Argentina \\
\hline \multicolumn{3}{|c|}{ Vicepresidentes SIRGAS } \\
\hline $\begin{array}{l}2002-2007 \\
2007-2011 \\
2011-2015\end{array}$ & $\begin{array}{l}\text { Eduardo Lauría } \\
\text { Laura Sánchez }\end{array}$ & $\begin{array}{l}\text { Instituto Geográfico Militar, Argentina } \\
\text { Deutsches Geodätisches Forschungsinstitut, } \\
\text { Alemania }\end{array}$ \\
\hline \multicolumn{3}{|c|}{ Presidentes del GT I } \\
\hline $\begin{array}{l}2008- \\
2007-2008\end{array}$ & $\begin{array}{l}\text { María Virginia Mackern } \\
\text { Sonia Maria Alves } \\
\text { Costa }\end{array}$ & $\begin{array}{l}\text { Universidad Nacional de Cuyo, Argentina } \\
\text { Instituto Brasileiro de Geografia e Estatística, } \\
\text { Brasil }\end{array}$ \\
\hline 2004-2007 & Claudio Brunini & Universidad Nacional de La Plata, Argentina \\
\hline 1993-2004 & Melvin Hoyer Romero & Universidad del Zulia, Venezuela \\
\hline \multicolumn{3}{|c|}{ Presidentes del GT II } \\
\hline $2008-$ & William Martínez Díaz & $\begin{array}{l}\text { Instituto Geográfico Agustín Codazzi, } \\
\text { Colombia }\end{array}$ \\
\hline $2007-2008$ & Tomás Marino Herrera & Universidad Nacional, Costa Rica \\
\hline $200-2007$ & Wilfredo Amaya Zelaya & $\begin{array}{l}\text { Instituto Geográfico y del Catastro Nacional, } \\
\text { El Salvador }\end{array}$ \\
\hline 2004-2005 & Antonio Hernández & $\begin{array}{l}\text { Instituto Nacional de Estadística Geogarafía e } \\
\text { Informática, México }\end{array}$ \\
\hline 1995-2004 & Rodrigo Barriga Vargas & Instituto Geográfico Militar, Chile \\
\hline 1993-1995 & Walter Subiza & Servicio Geográfico Militar, Uruguay \\
\hline \multicolumn{3}{|c|}{ Presidentes del GT III } \\
\hline 2013- & Sílvio Correia de Freitas & Universidade Federal do Paraná \\
\hline $2008-2013$ & Roberto Teixeira Luz & $\begin{array}{l}\text { Instituto Brasileiro de Geografia e Estatística, } \\
\text { Brasil }\end{array}$ \\
\hline $2007-2008$ & William Martínez Díaz & $\begin{array}{l}\text { Instituto Geográfico Agustín Codazzi } \\
\text { Colombia }\end{array}$ \\
\hline $2008-2007$ & Laura Sánchez & $\begin{array}{l}\text { Instituto Geográfico Agustín Codazzi, } \\
\text { Colombia }\end{array}$ \\
\hline $1997-2001$ & Roberto Texeira Luz & $\begin{array}{l}\text { Instituto Brasileiro de Geografia e Estatística, } \\
\text { Brasil }\end{array}$ \\
\hline
\end{tabular}


La primera versión tomó estado público a finales de 2002, fue hospedada por la institución a la que pertenecía el presidente de SIRGAS, por entonces Luiz Paulo Souto Fortes, del IBGE (Brasil), bajo cuya responsabilidad fue desarrollada y mantenida. Hacia finales de 2002 SIRGAS sanciona el estatuto que aún rige su vida institucional, el cual introduce la figura del vicepresidente a quien asigna, entre otras, la responsabilidad de mantener el portal. Este se traslada entonces del IBGE al IGM argentino y su mantenimiento queda bajo la responsabilidad de Eduardo Lauria, primer vicepresidente de SIRGAS. En 2007, la vicepresidencia de SIRGAS recae en Laura Sánchez, de DGFI (Alemania); desde entonces, el portal es hospedado por esa institución y su mantenimiento es llevado adelante por Laura Sánchez.

\section{Comentarios finales}

Además del respaldo científico y técnico brindados por la IAG y el IPGH de manera continuada desde la creación de SIRGAS en 1993, su apoyo se ha materializado de dos maneras complementarias e igualmente relevantes: mediante subsidios regulares, que SIRGAS ha aplicado casi exclusivamente a facilitar intercambios científicos y académicos que han beneficiado a numerosas instituciones en casi todos los países de América Latina y más recientemente del Caribe; y mediante un sostenido respaldo intelectual al accionar de SIRGAS frente a los institutos geográficos y otras agencias gubernamentales de la comunidad panamericana. Tales apoyos han resultado esenciales para convertir a SIRGAS en el principal foro de debate y en el principal agente de difusión de los avances de la Geodesia de América Latina y el Caribe (con reuniones anuales, escuelas y talleres que en promedio convocan a más de un centenar de participantes); siendo el principal articulador de la actividad geodésica de referencia en la región.

A través de sus grupos de trabajo, SIRGAS desarrolla en forma permanente una multiplicidad de actividades que abarcan: i) la materialización de un sistema de referencia vertical asociado al campo de gravedad terrestre, preciso y consistente a escala continental y global, lo cual requiere el análisis de las redes de nivelación y gravedad de todos los países latinoamericanos, conjuntamente con observaciones mareográficas y de altimetría satelital oceánica; ii) el apoyo al establecimiento de marcos de referencia nacionales vinculados a SIRGAS en los países que aún no lo han hecho y la promoción de la implementación de técnicas avanzadas de posicionamiento (por ejemplo, las denominadas de "tiempo real"); iii) la actualización permanente de las convenciones y modelos utilizados para elaborar los productos que SIRGAS pone a disposición de la comunidad; iv) estudios orientados a la comprensión del cambio global, tales como la producción de mapas de variables atmosféricas y el monitoreo del nivel del mar mediante mareógrafos controlados con GNSS; v) asistencia técnica permanente a una vasta comunidad allegada a SIRGAS y a la Organización de los Estados Americanos, a través del IPGH, para la resolu- 
ción de diferendos limítrofes; vi) participación en reuniones internacionales de la IUGG, de la IAG y de sus componentes para garantizar que los quehaceres de SIRGAS comulgan con los avances científicos de la Geodesia a nivel mundial. Todas estas actividades son de largo aliento y se desarrollan con apoyo del IPGH y de la IAG, de forma permanente, bajo la coordinación de su Comité Ejecutivo y con la participación, en los Grupos de Trabajo, de científicos y técnicos, no sólo de los 19 países de América Latina y el Caribe que lo conforman, sino también de entidades transcontinentales, en especial, el Deutches Geodätisches Forschungsinstitut (DGFI).

\section{Bibliografía}

Báez J.C., de Freitas S.C.R, Drewes, H., Dalazoana, R., Luz, R.T. (2007), "Deformations control for the Chilean part of the SIRGAS 2000 frame", Springer, IAG Symposia, vol. 130, pp. 660-664.

Brunini C., Sánchez L. (2012). "Geodetic activities in Latin America and The Caribbean: alwais IN", Coordinates, vol. VIII, Issue 6, June.

- (2013). "Geodetic Reference Frame for the Americas", GIM International, 3(27):26-31.

Brunini C., Moirano J.F., Mackern M.V. (2000). "Comparación entre los marcos de referencia POSGAR'94 y POSGAR'98”, Actas de la 20a Reunión de Geofísica y Geodesia, Mendoza, Argentina, 21-29 septiembre.

Brunini C., Meza A., Gende M., Azpilicueta F. (2008). "South American regional ionospheric maps computed by GESA: a pilot service in the framework of SIRGAS”, Advances in Space Research, doi:10.1016/j.asr.2007.08.041.

Brunini, C., Sánchez L., Drewes H., Costa S., Mackern V., Martínez W., Seemüller W., Da Silva A. (2012). "Improved Analysis Strategy and Accessibility of the SIRGAS Reference Frame", in Kenyon S., M.C. Pacino, U. Marti (eds.), "Geodesy for Planet Earth", IAG Symposia, No. 136, pp. 3-10.

Bruyninx, C., Altamimi Z., Becker M., Craymer M., Combrinck L., Combrink A., Dawson J., Dietrich R., Fernandes R., Govind R., Herring T., Kenyeres A., King R., Kreemer C., Lavallée D., Legrand J., Sánchez L., Sella G., Shen Z., Santamaría-Gómez A., Wöppelmann G. (2012). "A Dense Global Velocity Field Based on GNSS Observations: Preliminary Results", in Kenyon S., M.C. Pacino, U. Marti (eds.), "Geodesy for Planet Earth", IAG Symposia, No. 136, pp. 19-26.

Cadess H., Henneberg H., Palleja E., Pinch M. (1993). "Inter-American Geodetic Integration", Springer, IAG Symposia, vol. 111, pp. 3-15.

Cioce V., Hoyer M., Wildermann E., Royero G., Espinosa R., Méndez T. (2010). "Aprovechamiento de la insfraestructura observacional SIRGAS-CON en Venezuela para el seguimiento del vapor de agua troposférico", presentado en la Reunión SIRGAS 2010, noviembre 11 y 12, Lima, Perú. 
Costa S.M.A.C., Lima M.A.A. (2005). “Ajustamento da Rede Planimétrica Brasileira em SIRGAS2000”, IV Colóquio Brasileiro de Ciências Geodésicas, Curitiba, PR. IBGE, Brasil.

Costa S.M.A., Da Silva A.L., Vaz J.A. (2012). "Report on the SIRGAS-CON Combined Solution by IBGE Analysis Center", in Kenyon S., M.C. Pacino, U. Marti (eds.), "Geodesy for Planet Earth", IAG Symposia, No. 136, pp. 853-858.

Cruz Ramos O., Sánchez L. (2012). Efectos en el marco de referencia SIRGAS del terremoto del 7 de noviembre de 2012 en Guatemala, DGFI, Munich, Nov. 16, 2012.

Drewes H. (1995). "Sistema de Referencia Geocentrico para America del Sur", Proceedings IGS Workshop Densification of the IERS Terrestrial Reference Frame through regional GPS networks, A65-A73.

- (1999). "Report on the South American Geocentric Reference System (SIRGAS)", in O. Andersen (Ed.), Travaux International Association of Geodesy, No. 31, pp. 381-383.

. (2001). The unified height reference system for the Americas 7th UN Reg. Cart. Conf. for the Americas, E/CONF.93/INF.14.

- (2003). "Report 1999-2003 on the Geocentric Reference System for the Americas (SIRGAS)", Travaux International Association of Geodesy, vol. 32,2 pp.

. (2012). "How to Fix the Geodetic Datum for Reference Frames in Geosciences Applications?", in Kenyon S., M.C. Pacino, U. Marti (eds.), “Geodesy for Planet Earth", IAG Symposia, No. 136, pp. 657-664.

Drewes H., Fortes L.P.S, Hoyer M.J., Barriga R. (1997). Status report of the SIRGAS project IGS Annual Report, No. 1996, pp. 433-436.

Drewes H., Tremel H., Hernández J.N. (1998). “Adjustment of the new Venezuelan national GPS network within the SIRGAS reference frame", Springer; IAG Symposia; vol. 118, pp. 193-198.

Drewes H., Heidbach O. (2012). "The 2009 Horizontal Velocity Field for South America and the Caribbean", in Kenyon S., M.C. Pacino, U. Marti (eds.), "Geodesy for Planet Earth", IAG Symposia, No. 136, pp. 657-664.

Drewes H., Sánchez L., Blitzkow D., De Freitas S. (2002). "Scientific foundations of the SIRGAS vertical reference system", Springer; IAG Symposia, vol. 124, pp. 297-301.

Drewes H., Kaniuth K., Völksen C., Costa S.M.A., Fortes L.P.S. (2005). "Results of the SIRGAS campaign 2000 and coordinates variations with respect to the 1995 South American geocentric reference frame", Springer; IAG Symposia, vol. 128 , pp. 32-37. 
Echalar A., Sánchez L. (2010). “Ajuste del Marco de referencia Geodésico Nacional de Bolivia en SIRGAS: MARGEN-SIRGAS”, presentado en la Reunión SIRGAS 2010, noviembre 11 y 12, Lima, Perú.

Figueroa C., Amaya W., Sánchez L. (2010). "Integración de la red geodésica básica nacional de El Salvador a SIRGAS”, Revista Cartográfica, núms. 85-86, pp. 1934, Instituto Panamericano de Geografía e Historia, México.

Fortes L.P.S, Hoyer M.J., Subiza W., Drewes H. (1995). The SIRGAS project IGS Annual Report, 1994, pp. 247-250.

De Freitas S.R.C.; Medina A.S., De Lima S.R.S. (2002). "Associated problems to link South American vertical networks and possible approaches to face them", Springer; IAG Symposia, vol. 124, pp. 318-323.

Kaniuth K., Drewes H., Stuber K., Tremel H., Moirano J. (1998). "Results of the SIRGAS 95 GPS network processing at DGFI/I”, Springer; IAG Symposia, vol. 118, pp. 180-186.

Kaniuth K., Tremel H., Drewes H., Stuber K., Maturana R., Parra H. (2002a). "Processing of the SIRGAS 2000 GPS campaign at DGFI", Springer; IAG Symposia, vol. 124:312-317.

Kaniuth K., Mueller H., Seemüller W. (2002b). "Displacement of space geodetic observatory Arequipa due to recent earthquakes", Zeitschr. fuer Verm, No. 127, pp. 238-243.

Kumar M. (1993). "A geocentric approach for the new South American horizontal datum Proceedings", Int. Conf. on Cartography-Geodesy; Maracaibo, Venezuela, Inst. Astronomia y Geodesia; Madrid, No. 1, pp. 59-65.

Luz R. T. (2008). Estratégias para modernização da componente vertical do Sistema Geodésico Brasileiro e sua integração ao SIRGAS. Tese (Doutorado em Ciências Geodésicas) Universidade Federal do Paraná, Setor de Ciências da Terra, Curso de Pós-Graduação em Ciências Geodésicas, Curitiba, 207 f. Biblioteca Virtual da UFPR < http://www.cienciasgeodesicas.ufpr.br/teses/teses02.html $>$.

Luz R.T., Fortes L.P.S., Hoyer M.J., Drewes H. (2002). “The vertical reference frame for the Americas - The SIRGAS 2000 GPS campaign", Springer; IAG Symposia, vol. 124, pp. 302-305.

Martínez W., Sánchez, L. (2009). "Realization of the SIRGAS Reference Frame in Colombia”, Springer, IAG Symposia, vol. 134, pp. 185-190.

Moirano J., Brunini C., Drewes H., Kaniuth K. (1998). "Realization of a geodetic reference system in Argentina in connection with SIRGAS", Springer, IAG Symposia, vol. 118, pp. 199-204.

Sánchez L. (2004). Adopción del Marco Geocéntrico Nacional de Referencia MAGNA-SIRGAS como datum oficial de Colombia, Instituto Geográfico "Agustín Codazzi”, Bogotá, Colombia. 
(2007). "Definition and realization of the SIRGAS vertical reference system within a globally unified height system", Springer, IAG Symposia, vol. 130, pp. 638-645.

- (2008). "Approach for the establishment of a global vertical reference level", IAG Symposia, vol. 132, pp. 119-124, Springer, Berlin, Heidelberg.

. (2009). "Strategy to Establish a Global Vertical Reference System", Springer, IAG Symposia, vol. 134, pp. 273-278.

. (2012). "IGS Regional Network Associate Analysis Centre for SIRGAS (IGS RNAAC SIR) Report of activities 2011”, in Meindl, M., R. Dach, Y. Jean (Eds.), International GNSS Service, Technical Report 2011; Astronomical Institute, University of Bern, pp. 107-115.

Sánchez L., Brunini C. (2008). "SIRGAS: Basis for geosciences, geodata and navigation in Latin America", in Proceedings of the International Symposium on Global Navigation Satellite Systems, Space-based and Ground-based Augmentation Systems and Applications, Berlin, 11-14 November, Berlin Senate Department for Urban Development, pp. 37-39.

- (2009). "Achievements and Challenges of SIRGAS", Springer, IAG Symposia, vol. 134, pp. 161-166.

Sánchez L., Seemüller W., Krügel M. (2008). "Comparison and combination of the weekly solutions delivered by the SIRGAS Experimental Processing Centers", DGFI Report, No. 8o, Munich, 77 p.

Sánchez L., Seitz M. (2011). "Recent activities of the IGS Regional Network Associate Analysis Centre for SIRGAS (IGS RNAAC SIR)", DGFI Report, No. 87.

Sánchez L., Seemüller W., Drewes H. (2010). SIRGAS y el terremoto del 4 de abril de 210 en México. Reporte de cálculo. Centro de Análisis SIRGAS en el DGFI.

Sánchez L., Brunini C., Mackern V., Martínez W., Luz R.T. (2011). "SIRGAS: the geocentric reference frame of the Americas", Proceedings of the International Symposium on Global Navigation Satellite Systems, Space-Based and GroundBased Augmentation Systems and Applications 2010, Brussels, Belgium, November 29-30, 2010, Berlin Senate Department for Urban Development, pp. 21-25.

Sánchez L., Seemüller W., Seitz M. (2012). "Combination of the Weekly Solutions Delivered by the SIRGAS Processing Centres for the SIRGAS-CON Reference Frame", in Kenyon S., M.C. Pacino, U. Marti (eds.), "Geodesy for Planet Earth", IAG Symposia, 136, pp. 845-852.

Sánchez L., Seemüller W., Drewes H., Mateo L., González G., Da Silva A., Pampillón J., Martínez W., Cioce V., Cisneros D., Cimbaro S. (2013). "Long-Term Stability of the SIRGAS Reference Frame and Episodic Station Movements Caused by the Seismic Activity in the SIRGAS Region", in Altamimi Z. and 
Collilieux X (eds.), Reference Frames for Applications in Geosciences, IAG Symposia, 138, pp. 153-161, Springer Berlin Heidelberg.

Seemüller W. (2009). "The Position and Velocity Solution DGF06P01 for SIRGAS”, Springer, IAG Symposia; vol. 134, pp. 167-172.

Seemüller W., Drewes, H. (1998a). "The IGS Regional Associate Analysis Center for South America at DGFI/I”, Springer, IAG Symposia, vol. 118, pp. 211-215. . (1998b). "Annual report 1997 of the RNAAC SIRGAS", IGS 1997 Technical Reports, 173-174, IGS CB, JPL Pasadena.

. (1999). "Annual Report 1998 of RNAAC SIRGAS”, IGS 1998 Technical Reports, pp. 133-134, IGS CB, JPL Pasadena.

- (2000). "Annual Report 1999 of RNAAC SIRGAS”, IGS 1999 Technical Reports, IGS CB, JPL Pasadena.

- (2002). Annual Report 2000 of RNAAC SIR IGS Techn. Rep., 2000; JPL Publ. 02-012:141-144.

Seemüller W., Kaniuth K., Drewes H. (2002). "Velocity estimates of IGS RNAAC SIRGAS stations", Springer, IAG Symposia, vol. 124, pp. 7-10.

- (2004). "Station positions and velocities of the IGS regional network for SIRGAS”, DGFI Report, núm. 76.

Seemüller W., Krügel M., Sánchez L. (2008). “The position and velocity solution DGF08P01 of the IGS Regional Network Associate Analysis Centre for SIRGAS (IGS-RNAAC-SIR)", DGFI Report No. 29, Munich, p. 110.

Seemüller W., Seitz M., Sánchez L., Drewes H. (2009). “The position and velocity solution SIR09P01 of the IGS Regional Network Associate Analysis Centre for SIRGAS (IGS RNAAC SIR)", DGFI Report No. 85, Munich, p. 112.

Seemüller W., Sánchez L., Seitz M., Drewes H. (2010). “The position and velocity solution SIR10P01 of the IGS Regional Network Associate Analysis Centre for SIRGAS (IGS RNAAC SIR)", DGFI Report No. 86.

. (2012). "The new Multi-year Position and Velocity Solution SIR09P01 of the IGS Regional Network Associate Analysis Centre (IGS RNAAC SIR)", in Kenyon S., M.C. Pacino, U. Marti (Eds.), "Geodesy for Planet Earth", IAG Symposia, 136, pp. 877-884.

SIRGAS Project Committee (1997). "SIRGAS Final Report", Working Groups I and II IBGE, Rio de Janeiro, p. 96.

Tremel H., Sánchez L., Drewes H. (2001). "Procesamiento de la red GPS básica de Colombia: Marco Geocéntrico Nacional - MAGNA”, Revista Cartográfica IPGH, núm. 73, pp. 7-23.

Zurita P., Cisneros D., Bayas M., Amores M. (2010). “Adopción de SIRGAS como nuevo marco de referencia para el Ecuador”, Presentado en la Reunión SIRGAS 2010, noviembre 11 y 12, Lima, Perú. 